Draft Version November 28, 2018

Preprint typeset using $\mathrm{LAT}_{\mathrm{E}} \mathrm{X}$ style emulateapj v. 08/22/09

\title{
M31 TRANSVERSE VELOCITY AND LOCAL GROUP MASS FROM SATELLITE KINEMATICS
}

\author{
RoEland P. VAN DER MAREL \\ Space Telescope Science Institute, 3700 San Martin Drive, Baltimore, MD 21218
}

\author{
Puragra Guhathakurta \\ UCO/Lick Observatory, Department of Astronomy and Astrophysics, University of California at Santa Cruz, 1156 High Street, Santa \\ Cruz, CA 95064 \\ Draft version November 28, 2018
}

\begin{abstract}
We present several different statistical methods to determine the transverse velocity vector of M31. The underlying assumptions are that the M31 satellites on average follow the motion of M31 through space, and that the galaxies in the outer parts of the Local Group on average follow the motion of the Local Group barycenter through space. We apply the methods to the line-of-sight velocities of 17 M31 satellites, to the proper motions of the 2 satellites M33 and IC 10, and to the line-of-sight velocities of 5 galaxies near the Local Group turn-around radius, respectively. This yields 4 independent but mutually consistent determinations of the heliocentric M31 transverse velocities in the West and North directions, with weighted averages $\left\langle v_{W}\right\rangle=-78 \pm 41 \mathrm{~km} \mathrm{~s}^{-1}$ and $\left\langle v_{N}\right\rangle=-38 \pm 34 \mathrm{~km} \mathrm{~s}^{-1}$. The

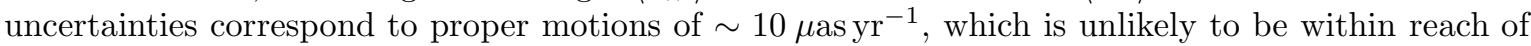
direct observational verification within the next decade. The Galactocentric tangential velocity of M31 is $42 \mathrm{~km} \mathrm{~s}^{-1}$, with $1 \sigma$ confidence interval $V_{\tan } \leq 56 \mathrm{~km} \mathrm{~s}^{-1}$. The implied M31-Milky Way orbit is bound if the total Local Group mass $M$ exceeds $1.72_{-0.25}^{+0.26} \times 10^{12} \mathrm{M}_{\odot}$. If the orbit is indeed bound, then the timing argument combined with the known age of the Universe implies that $M=5.58_{-0.72}^{+0.85} \times 10^{12} \mathrm{M}_{\odot}$. This is on the high end of the allowed mass range suggested by cosmologically motivated models for the individual structure and dynamics of M31 and the Milky Way, respectively. It is therefore possible that the timing mass is an overestimate of the true mass, especially if one takes into account recent results from the Millennium Simulation that show that there is also a theoretical uncertainty of $41 \%$ (Gaussian dispersion) in timing mass estimates. The M31 transverse velocity implies that M33 is in a tightly bound orbit around M31. This may have led to some tidal deformation of M33. It will be worthwhile to search for observational evidence of this.

Subject headings: galaxies: kinematics and dynamics — Local Group — M31.
\end{abstract}

\section{INTRODUCTION}

The Local Group is dominated by two spiral galaxies, M31 and the Milky Way. These galaxies have comparable properties, with M31 generally believed to be slightly more massive (e.g., Klypin, Zhao \& Somerville 2002). The next most luminous galaxies, M33 and the Large Magellanic Cloud, are some 10 times fainter (e.g., van den Bergh 2000). The dynamics and future of the Local Group are therefore determined primarily by the relative velocity of M31 with respect to the Milky Way. Unfortunately, this velocity is poorly known. The lineof-sight velocity of M31 can be measured extremely accurately using the Doppler shift of a large variety of tracers. However, even after a century of careful attempts (starting with, e.g., Barnard 1917) still no useful proper motion measurement exists to constrain the transverse velocity. This limits our ability to answer several fundamental questions. For example, do M31 and the Milky Way indeed form a bound system, as is usually assumed (e.g., van den Bergh 1971)? What is the exact mass of the Local Group implied by the so-called timing argument (e.g., Kahn \& Woltjer 1959; Kroeker \& Carlberg 1991; Lynden-Bell 1999)? What is the expected future evolution of the M31-Milky Way system (e.g., Cox \& Loeb 2007)? And how has the structure of M33 been influenced by possible interaction with M31 (e.g., Loeb et al. 2005; hereafter L05)?

In the present paper we show that it is possible to obtain a statistical determination of the transverse velocity of M31 using the observed velocities of its satellites. The analysis assumes that on average the satellites follow the motion of M31 through space, with some velocity dispersion. The ensemble of line-of-sight velocities, and the individual proper motions available for selected galaxies, then yield independent estimates of the M31 velocity. We also revisit the method previously explored by Einasto \& Lynden-Bell (1982), which is based on the assumption that the galaxies in the outer parts of the Local Group follow the motion of the Local Group barycenter through space. We apply the different methods to the currently available data for the relevant Local Group galaxies, and we combine the results to obtain an accurate determination of the M31 transverse velocity. We then use this determination to address the aforementioned questions.

The structure of the paper is as follows. Section 2 discusses the constraints on the M31 transverse velocity, based on: the line-of-sight velocities of an ensemble of 17 M31 satellites (Section 2.1); the recent high accuracy proper motion determinations of the M31 satellites M33 and IC 10 by Brunthaler et al. $(2005,2007)$ from VLBI observations of water masers (Section 2.2); and the lineof-sight velocities of an ensemble of galaxies in the outer 
parts of the Local group (Section 2.3). The different constraints are compared and combined in Section 2.4. Section 3 discusses the implications of the inferred M31 velocity for the relative orbit of M31 and the Milky Way (Section 3.1) and for the relative orbit of M31 and M33 (Section 3.2). Section 4 discusses how the results for the M31 transverse velocity (Section 4.1), the total mass of the Local group as implied by the timing argument (Section 4.2), and the Local Group turn-around radius (Section 4.3) compare with theoretical predictions and other observational studies. Section 5 presents the conclusions of our work.

In the analysis below we use several coordinate systems. Observational systems have their three principal axes aligned with the line-of-sight, West, and North directions, respectively, for a given position on the sky. We also use a Galactocentric coordinate system centered on the Milky Way and a barycentric coordinate system centered on the Local Group barycenter. The analysis requires transformations between the positions and velocities in these systems. Many of the necessary notations and derivations can be found in van der Marel et al. (2002; which presents a study of the kinematics of the Large Magellanic Cloud), and are given here without further reference. Heliocentric velocities are generally denoted with a vector $\vec{v}$, Galactocentric velocities with a vector $\vec{V}$, and Local-Group barycentric velocities with a vector $\vec{U}$. Velocities and proper motions in the observational systems are generally heliocentric (i.e., not corrected for the reflex motion of the Sun), unless stated otherwise.

\section{THE M31 TRANSVERSE VELOCITY}

\subsection{Constraints from Line-of-Sight Velocities of M31 Satellites}

The velocity vector of an M31 satellite galaxy can be written as the sum of the M31 velocity vector, and a peculiar velocity

$$
\vec{v}_{\mathrm{sat}}=\vec{v}_{\mathrm{M} 31}+\vec{v}_{\mathrm{pec}} .
$$

We assume that any one-dimensional component of $\vec{v}_{\text {pec }}$ is a random Gaussian deviate with dispersion $\sigma$. More formally, this is true if the velocity distribution of the satellites is both isotropic and isothermal. The first assumption finds some support from studies of galaxy satellite systems (e.g., Kochanek 1996) and clusters of galaxies (e.g., van der Marel et al. 2000). The second assumption is reasonable in view of the fact that the gravitational potentials of dark halos are approximately logarithmic. Either way, in the present analysis there is only a very weak dependence on the accuracy of these assumptions (by contrast to studies of the mass distribution of M31; e.g., Evans et al. 2000).

The velocity vector of M31 can be described by quantities $v_{\text {sys }}, v_{t}$ and $\Theta_{t}$, where $v_{\text {sys }}$ is the line-of-sight velocity, $v_{t}$ is the transverse velocity, and $\Theta_{t}$ is the position angle of the transverse motion on the sky. The velocities in the directions of West and North are

$$
v_{W} \equiv v_{t} \cos \left(\Theta_{t}+90^{\circ}\right), \quad v_{N} \equiv v_{t} \sin \left(\Theta_{t}+90^{\circ}\right) .
$$

The position of a satellite on the sky can be described by the angles $(\rho, \Phi)$, where $\rho$ is the angular distance from
TABLE 1

\begin{tabular}{|c|c|c|c|c|}
\hline $\begin{array}{c}\text { Name } \\
(1)\end{array}$ & $\begin{array}{c}\text { Type } \\
(2)\end{array}$ & $\begin{array}{c}\rho \\
\text { deg } \\
(3)\end{array}$ & $\begin{array}{c}\Phi \\
\text { deg } \\
(4)\end{array}$ & $\frac{\mathrm{km} \mathrm{s}^{-1}}{(5)}$ \\
\hline M31 & $\mathrm{Sb}$ I-II & 0.00 & & $-301 \pm 1$ \\
\hline M32 & E2 & 0.40 & -179.10 & $-205 \pm 3$ \\
\hline NGC 205 & dSph & 0.60 & -46.61 & $-244 \pm 3$ \\
\hline And IX & $\mathrm{dSph}$ & 2.69 & 43.32 & $-211 \pm 3$ \\
\hline And I & dSph & 3.27 & 169.84 & $-380 \pm 2$ \\
\hline And III & $\mathrm{dSph}$ & 4.97 & -163.13 & $-355 \pm 10$ \\
\hline And X & dSph & 5.62 & 48.96 & $-164 \pm 3$ \\
\hline NGC 185 & dSph/dE3 & 7.09 & -5.07 & $-202 \pm 7$ \\
\hline NGC 147 & $\mathrm{dSph} / \mathrm{dE} 5$ & 7.43 & -12.29 & $-193 \pm 3$ \\
\hline And V & dSph & 8.03 & 35.31 & $-403 \pm 4$ \\
\hline And II & dSph & 10.31 & 136.82 & $-188 \pm 3$ \\
\hline M33 & Sc II-III & 14.78 & 131.78 & $-180 \pm 1$ \\
\hline And VII/Cas & dSph & 16.17 & -47.93 & $-307 \pm 2$ \\
\hline IC 10 & dIrr & 18.37 & -9.11 & $-344 \pm 5$ \\
\hline And VI/Peg & dSph & 19.76 & -143.64 & $-354 \pm 3$ \\
\hline LGS 3/Pisces & $\mathrm{dIrr} / \mathrm{dSph}$ & 19.89 & 165.44 & $-286 \pm 4$ \\
\hline Pegasus & $\mathrm{dIrr} / \mathrm{dSph}$ & 31.01 & -143.37 & $-182 \pm 2$ \\
\hline IC 1613 & dIrr V & 39.44 & 171.30 & $-232 \pm 5$ \\
\hline
\end{tabular}

M31 Satellite Galaxy Sample

NotE. - The sample of M31 satellites used in the modeling of Section 2.1. M31 itself is listed on the first line for comparison. Column (1) lists the galaxy name and column (2) its type. Columns (3) and (4) define the position on the sky: $\rho$ is the angular distance from M31 and $\Phi$ is the position angle with respect to M31 measured from North over East. These angles were calculated from the sky positions (RA,DEC) as in van der Marel et al. (2002). The satellites in the table are sorted by their value of $\rho$. Column (5) lists the heliocentric line-of-sight velocity and its error. Velocities and sky positions (RA,DEC) for most satellites were obtained from the compilation of Evans et al. (2000), except for the satellites And IX and X which had not yet been discovered in 2000. For those we used the velocity measurements and (RA,DEC) given by Chapman et al. (2005) and Kalirai et al. (2007), respectively.

M31 and $\Phi$ is the position angle with respect to M31 measured from North over East (as defined in van der Marel \& Cioni 2001; van der Marel et al. 2002). Because satellites are not located on the same position on the sky as M31, the vector $\vec{v}_{\mathrm{M} 31}$ has a different decomposition in line-of-sight and transverse components than it does for M31. More specifically, the line-of-sight velocity of a satellite is

$$
v_{\text {sat,los }}=v_{\text {sys }} \cos \rho+v_{t} \sin \rho \cos \left(\Phi-\Theta_{t}\right)+v_{\text {pec,los }} .
$$

The factor $\cos \rho$ in the first term indicates that only a fraction of $v_{\text {sys }}$ is seen along the line-of-sight. The second term is an apparent solid body rotation component in the $v_{\text {sat,los }}$ velocity field on the sky, with amplitude $v_{t} \sin \rho$ and kinematic major axis along position angle $\Theta_{t}$. The last term merely adds a scatter $\sigma$ on top of the velocity field defined by the first two terms.

It follows from equation (3) that the transverse velocity of M31 affects the line-of-sight velocities of its satellites. Therefore, a study of the line-of-sight velocities of the satellites can constrain the M31 transverse velocity. To this end we compiled the sample in Table 1, which consists of M31 satellites with known velocities. The sample is based on that used by Evans et al. (2000), but with ad- 
dition of the more recently discovered Andromeda dwarf satellites And IX and X. The satellites And XI and XIII (Martin et al. 2006, who also reports the finding of an unusually distant globular cluster) and And XV and XVI (Ibata et al. 2007) are not included because no line-ofsight velocity measurements have yet been reported for them. We fitted equation (31) to these data by determining the values of $\left(v_{\mathrm{sys}}, v_{W}, v_{N}\right)$ that minimize the scatter in $v_{\text {pec,los. }}$. The resulting scatter is the dispersion $\sigma$ of the satellite population. After the best-fitting model was identified, we calculated error bars on the fitted parameters using Monte-Carlo simulations. Many different pseudo data sets were created with the same satellites at the same positions, but with velocities drawn from the best-fit model, with $v_{\text {pec,los }}$ drawn as a random Gaussian deviate with dispersion $\sigma$. The pseudo data sets were then analyzed similarly as the real data set. The dispersions in the inferred model parameters are a measure of their formal 1- $\sigma$ error bars.

The modeling procedure yields $v_{\text {sys }}=-270 \pm 19 \mathrm{~km} \mathrm{~s}^{-1}$, $v_{W}=-136 \pm 148 \mathrm{~km} \mathrm{~s}^{-1}, v_{N}=-5 \pm 75 \mathrm{~km} \mathrm{~s}^{-1}$, and $\sigma=76 \pm 13 \mathrm{~km} \mathrm{~s}^{-1}$ (see also Table 3 below). The data and representative predictions of the best-fitting model are shown in Figure 1. Color-coding indicates the distance from M31. The inferred transverse velocity corresponds to sinusoidal variations that are less than the observed scatter in the data. No sinusoidal variation with angle is discernible in the data by eye; indeed, the case of zero transverse velocity (amplitude zero for the sinusoidal variations) is also statistically consistent with the data. However, this is not a null result. Large transverse velocities of hundreds of $\mathrm{km} \mathrm{s}^{-1}$ would have induced large sinusoidal variations that are not seen in the data. Such transverse velocities are therefore ruled out. We note that we could also have kept $v_{\text {sys }}$ fixed in the fit at the observed value of $v_{\text {sys }}=-301 \pm 1 \mathrm{~km} \mathrm{~s}^{-1}$ (Courteau \& van den Bergh 1999). We verified that such a fit yields verify similar results, namely $v_{W}=-123 \pm 159 \mathrm{~km} \mathrm{~s}^{-1}$ and $v_{N}=-33 \pm 79 \mathrm{~km} \mathrm{~s}^{-1}$, which is well within the uncertainties quoted above.

Our analysis does not assume that the satellites are necessarily bound to M31. For M33, orbit calculations do suggest that it probably is bound (see figure 3 of Loeb et al. 2005), but this has not been established with confidence for most of the other satellites. We decided not to include the recently discovered $\mathrm{dSph}$ galaxies And XII and And XIV in our sample. They lie at similar position angles, $\Phi=171.94^{\circ}$ and $170.49^{\circ}$, respectively, and at distances $\rho=6.96^{\circ}$ and $11.71^{\circ}$, that are not atypical for the rest of our sample. However, their velocities of $-556 \pm 5$ and $-478 \pm 5 \mathrm{~km} \mathrm{~s}^{-1}$, respectively, are $3.3 \sigma$ and $2.3 \sigma$ away from the observed velocity of M31. It has been suggested that they form a system that is falling into M31 for the first time (Chapman et al. 2007; Majewski et al. 2007), which would not make them useful additions to our analysis. As a test we did repeat our analysis with these galaxies included in the sample. This yielded $v_{\text {sys }}=-296 \pm 27 \mathrm{~km} \mathrm{~s}^{-1}, v_{W}=-85 \pm 200 \mathrm{~km} \mathrm{~s}^{-1}$, $v_{N}=41 \pm 108 \mathrm{~km} \mathrm{~s}^{-1}$, and $\sigma=106 \pm 18 \mathrm{~km} \mathrm{~s}^{-1}$. This is consistent with the result for our main sample to within the uncertainties. This illustrates that the results are fairly robust against the inclusion or removal of individual galaxies. By contrast, modeling of the parent galaxy

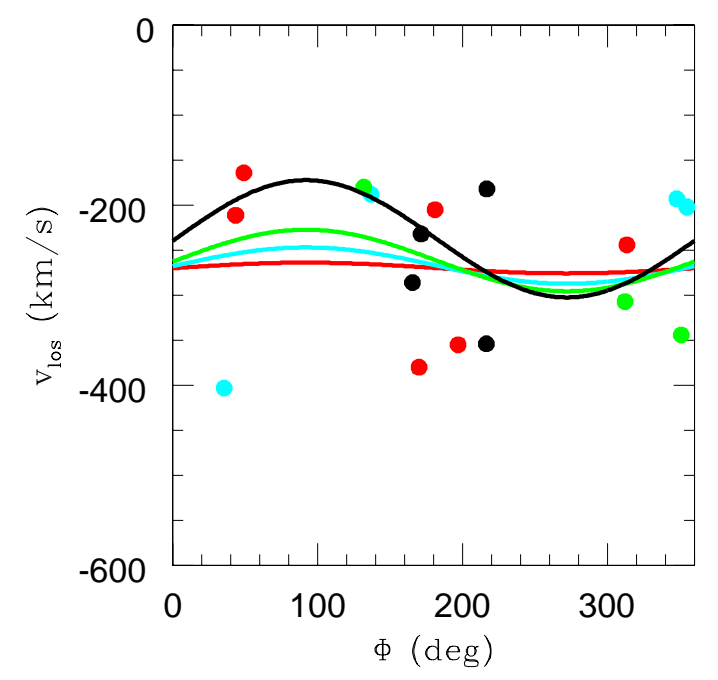

FIG. 1.- Comparison of heliocentric line-of-sight velocity data of M31 satellites and the predictions of equation (3), for the bestfit values of the heliocentric M31 transverse velocity $\left(v_{W}, v_{N}\right)=$ $(-136,-5) \mathrm{km} \mathrm{s}^{-1}$. The curves show the predictions as function of position angle $\Phi$, for angular distances from M31 of $\rho=3^{\circ}$ (red), $\rho=9^{\circ}$ (cyan), $\rho=15^{\circ}$ (green), $\rho=29^{\circ}$ (black). Data points are from Table 1 They are color coded based on bins in angular distance $\rho$, from $0-6^{\circ}$ (red), $6-12^{\circ}$ (cyan), $12-18^{\circ}$ (green), and 18$40^{\circ}$ (black). Predictions with zero transverse velocity (amplitude zero for the sinusoidal variations) are also statistically consistent with the data. However, a large transverse velocity for M31 of hundreds of $\mathrm{km} \mathrm{s}^{-1}$ (corresponding to sinusoidal variations of much larger amplitude than show here) are ruled out.

mass based on satellites can be quite sensitive to assumptions about the bound state of individual galaxies (e.g., Kochanek 1996).

\subsection{Constraints from Proper Motions of M31 Satellites}

Water masers can be observed at high spatial resolution with VLBI techniques. This makes them a valuable tool for proper motion studies of Local Group galaxies. Brunthaler et al. $(2005,2007)$ recently determined the proper motions for two galaxies in the M31 group, namely M33 and IC 10. Unfortunately, no water masers have yet been found in M31 and, at present detection limits, may be none should be expected (Brunthaler et al. 2006). So the direct application of this technique to M31 may not be possible in the foreseeable future. However, the measurements for M33 and IC 10 can be used to constrain the transverse velocity of M31 indirectly. Equation (1) implies that the unknown velocity vector of M31 can be estimated from the known velocity vector of a satellite as $\vec{v}_{M 31}=\vec{v}_{\text {sat }}-\vec{v}_{\text {pec }}$. Since the peculiar velocity is unknown, it acts as a Gaussian uncertainty of size $\sigma$ in each velocity component.

In analogy with equation (3), one can write for the transverse velocity components of the satellite

$$
\begin{aligned}
& v_{\text {sat }, 2}=-v_{\text {sys }} \sin \rho+v_{t} \cos \rho \cos \left(\Phi-\Theta_{t}\right)+v_{\text {pec }, 2}, \\
& v_{\text {sat }, 3}=-v_{t} \sin \left(\Phi-\Theta_{t}\right)+v_{\text {pec }, 3 .}
\end{aligned}
$$

Here the unit vectors 2 and 3 on the plane of the sky are related to the directions of West and North, all at the position of the satellite, according to a rotation

$$
\left(\begin{array}{c}
v_{\mathrm{sat}, W} \\
v_{\mathrm{sat}, N}
\end{array}\right)=\left(\begin{array}{cc}
-\sin \Gamma & -\cos \Gamma \\
\cos \Gamma & -\sin \Gamma
\end{array}\right)\left(\begin{array}{l}
v_{\mathrm{sat}, 2} \\
v_{\mathrm{sat}, 3}
\end{array}\right),
$$


where the rotation angle $\Gamma$ is determined by

$$
\begin{aligned}
\cos \Gamma & =\left[\sin \delta \cos \delta_{0} \cos \left(\alpha-\alpha_{0}\right)-\cos \delta \sin \delta_{0}\right] / \sin \rho, \\
\sin \Gamma & =\left[\cos \delta_{0} \sin \left(\alpha-\alpha_{0}\right)\right] / \sin \rho .
\end{aligned}
$$

Here $(\alpha, \delta)$ are the RA and DEC of the satellite, and $\left(\alpha_{0}, \delta_{0}\right)$ are the RA and DEC of M31 (i.e., the position with $\rho=0$ ).

Given values of $\left(v_{\mathrm{sat}, \mathrm{los}}, v_{\mathrm{sat}, W}, v_{\mathrm{sat}, N}\right)$, the equations (2)- (6) uniquely constrain the three unknown components $\left(v_{\mathrm{sys}}, v_{W}, v_{N}\right)$ of the M31 velocity vector. We solve these equations for each of the two satellites M33 and IC 10. We take $v_{\text {sat,los }}$ from Table 1 To obtain $v_{\text {sat,W }}$ and $v_{\text {sat,N }}$ we write each velocity component as $v=\left(0.0047404 D \mu_{\mathrm{obs}}\right)-\delta v_{\text {rot }}$, where $\mu_{\text {obs }}$ is the observed proper motion in $\operatorname{assyr}^{-1}, D$ is the satellite distance in $\mathrm{kpc}$, and $\delta v_{\text {rot }}$ is a correction for the internal rotation of the galaxy under study. We take $D=794 \pm 23 \mathrm{kpc}$ for M33 (McConnachie et al. 2004) and $D=660 \pm 65 \mathrm{kpc}$ for IC 10 (Evans et al. 2000). The other quantities follow from Brunthaler et al. (2005, 2007): $\left(\mu_{\mathrm{obs}, \mathrm{W}}, \mu_{\mathrm{obs}, \mathrm{N}}\right)=(-4.7 \pm 3.2,-14.1 \pm 6.4) \mu \mathrm{as} \mathrm{yr}^{-1}$ for M33; $\left(\mu_{\mathrm{obs}, \mathrm{W}}, \mu_{\mathrm{obs}, \mathrm{N}}\right)=(-6.0 \pm 5.0,23.0 \pm 5.0) \mu \mathrm{as} \mathrm{yr}^{-1}$ for IC10; $\left(\delta v_{\text {rot }, \mathrm{W}}, \delta v_{\text {rot }, \mathrm{N}}\right)=(70 \pm 23,-81 \pm 23) \mathrm{km} \mathrm{s}^{-1}$ for M33; and $\left(\delta v_{\text {rot }, \mathrm{W}}, \delta v_{\text {rot, } \mathrm{N}}\right)=(-25 \pm 19,9 \pm 19) \mathrm{kms}^{-1}$ for IC 10. We do not include the Brunthaler et al. corrections for the reflex motion of the Sun, since we deal with that issue separately in Section 3.1. We add Gaussian random deviates in our calculations to reflect the uncertainties. We take each component of $\vec{v}_{\text {pec }}$ to be a Gaussian random deviate with dispersion $\sigma=76 \mathrm{kms}^{-1}$, as determined in Section 2.1. For each combination of $\left(v_{\text {sat,los }}, v_{\text {sat, } \mathrm{W}}, v_{\text {sat, } \mathrm{N}}\right)$ we solve the equations to obtain $\left(v_{\text {sys }}, v_{W}, v_{N}\right)$ and we repeat this in Monte-Carlo fashion. We adopt the average and dispersion of the results as our final estimate for the M31 velocity vector and its error. Using M33, we obtain the following estimates for M31: $v_{\text {sys }}=-183 \pm 76 \mathrm{kms}^{-1}, v_{W}=-48 \pm 80 \mathrm{~km} \mathrm{~s}^{-1}$, $v_{N}=71 \pm 84 \mathrm{~km} \mathrm{~s}^{-1}$. Using IC 10 , we obtain the following estimates for M31: $v_{\text {sys }}=-346 \pm 76 \mathrm{~km} \mathrm{~s}^{-1}$, $v_{W}=-16 \pm 80 \mathrm{~km} \mathrm{~s}^{-1}, v_{N}=-47 \pm 81 \mathrm{~km} \mathrm{~s}^{-1}$ (see also Table 3 below).

\subsection{Constraints from Line-of-Sight Velocities of Outer Local Group Galaxies}

The Local Group contains not only the virialized subgroups of galaxies surrounding the Milky Way and M31, but also a number of unattached galaxies that populate the outer regions of the Local Group. On average, these galaxies are expected to follow the motion of the Local Group barycenter through space. Their heliocentric lineof-sight velocity vectors, averaged in a three-dimensional sense, therefore statistically equal the reflex motion of the Sun with respect to the Local Group barycenter. Since the velocity of the barycenter is itself determined by the relative velocity of M31 with respect to the Milky Way, this yields a determination of the latter. Variations of this method have been applied in the past by, e.g., Yahil, Tammann, \& Sandage (1977), Lynden-Bell \& Lin (1977) and Einasto \& Lynden-Bell (1982). It is now worthwhile to revisit this method, since available information on the membership and distances of Local Group galaxies has evolved significantly in the past decades.
We adopt a Cartesian coordinate system $(X, Y, Z)$, with the origin at the Galactic Center, the $Z$-axis pointing towards the Galactic North Pole, the $X$-axis pointing in the direction from the sun to the Galactic Center, and the $Y$-axis pointing in the direction of the sun's Galactic Rotation. For the set of outer Local Group galaxies $i=1, \ldots, N$ we calculate the unit vector $\vec{r}_{i}$ in the direction of each galaxy. If the velocity vector of the Sun with respect to the Local Group barycenter is $\vec{U}_{\odot}$, then one has

$$
\sum_{i}\left(v_{\operatorname{los}, i}+\left[\vec{U}_{\odot} \cdot \vec{r}_{i}\right]\right) \vec{r}_{i}=0
$$

where $v_{\mathrm{los}, i}$ is the heliocentric line-of-sight velocity for each galaxy. This can be written as a $3 \times 3$ matrix equation for the components of the vector $\vec{U}_{\odot}$. The bestfit values and their formal errors are easily obtained using standard techniques (Einasto \& Lynden-Bell 1982). Once this solution is obtained one can calculate the velocity of the Milky Way with respect to the Local Group Barycenter, $\vec{U}_{\mathrm{MW}}=\vec{U}_{\odot}-\vec{V}_{\odot}$. Here $\vec{V}_{\odot}$ is the velocity vector of the Sun in the Galactocentric rest frame. For the circular velocity of the Local Standard of Rest (LSR) we use the standard IAU value $V_{0}=220 \mathrm{~km} \mathrm{~s}^{-1}$ (Kerr \& Lynden-Bell 1986), to which we assign an uncertainty $10 \mathrm{~km} \mathrm{~s}^{-1}$ (none of our results depend sensitively on this quantity). For the residual velocity of the Sun with respect to the LSR we adopt the values of Dehnen \& Binney (1998). If we assume that all of the mass of the Local Group resides in the Milky Way and M31, then the barycenter is simply the mass-weighted average of their position vectors. This implies in the Galactocentric rest frame that the velocity of M31 is $\vec{V}_{\mathrm{M} 31}=-\vec{U}_{\mathrm{MW}} / f_{\mathrm{M} 31}$, where $f_{\mathrm{M} 31} \equiv M_{\mathrm{M} 31} /\left(M_{\mathrm{M} 31}+M_{\mathrm{MW}}\right)$. The heliocentric velocity of M31 is therefore $\vec{v}_{\mathrm{M} 31}=\vec{V}_{\mathrm{M} 31}-\vec{V}_{\odot}$. After substitution of the previous equations this yields

$$
\vec{v}_{\mathrm{M} 31}=-\vec{U}_{\odot} / f_{\mathrm{M} 31}+\vec{V}_{\odot}\left[\left(1 / f_{\mathrm{M} 31}\right)-1\right] .
$$

This heliocentric vector can be decomposed into components along the line-of-sight and in the West and North directions following the methodology of van der Marel et al. (2002).

Application of equation (8) requires that we assume a value for $f_{\mathrm{M} 31}$. Einasto \& Lynden-Bell (1982) used the Tully-Fisher relation to constrain this quantity, and built this constraint directly into their matrix solution for $\vec{U}_{\odot}$. However, the mass ratio of M31 and the Milky Way isn't actually all that well known observationally, and different arguments for estimating it have yielded different results. Our aim here is to constrain $\vec{v}_{\mathrm{M} 31}$ from observational data, while imprinting a minimum amount of theoretical prejudice into the result. So we simply assume that $f_{\mathrm{M} 31}$ is homogeneously distributed between 0.39 and 0.67 (i.e., $M_{\mathrm{M} 31} / M_{\mathrm{MW}}=0.8-2.0$ ). This encompasses most of the values that have been quoted in the literature (see discussion in Section 4.2). We then solve equation (8) in Monte-Carlo fashion, while simultaneously adding in the random errors in $\vec{U}_{\odot}$ and $\vec{V}_{\odot}$. This yields both the best fit result for $\vec{v}_{\mathrm{M} 31}$ and its statistical uncertainties.

We base our analysis on the sample of Local Group dwarf galaxies (there are no giant galaxies in the outer 
TABLE 2

Outer local Group Galaxy Sample

\begin{tabular}{|c|c|c|c|c|c|c|}
\hline $\begin{array}{c}\text { Name } \\
(1)\end{array}$ & $\begin{array}{l}\text { Type } \\
(2)\end{array}$ & $\begin{array}{l}\text { RA } \\
\text { deg } \\
(3)\end{array}$ & $\begin{array}{c}\text { DEC } \\
\text { deg } \\
(4)\end{array}$ & $\begin{array}{c}v_{\operatorname{los}} \\
\mathrm{km} \mathrm{s}^{-1} \\
(5)\end{array}$ & $\begin{array}{c}D \\
\mathrm{kpc} \\
(6)\end{array}$ & $\begin{array}{c}D_{\text {bary }} \\
\text { kpc } \\
(7)\end{array}$ \\
\hline $\begin{array}{l}\text { WLM } \\
\text { Aquarius/DD0210 } \\
\text { Leo A } \\
\text { Tucana } \\
\text { Sag DIG }\end{array}$ & $\begin{array}{l}\text { dIrr IV-V } \\
\text { dIrr/dSph } \\
\text { dIrr V } \\
\text { dSph } \\
\text { dIrr V }\end{array}$ & $\begin{array}{r}0.49234 \\
311.71585 \\
149.86025 \\
340.45667 \\
292.49573\end{array}$ & $\begin{array}{r}-15.46093 \\
-12.84792 \\
30.74639 \\
-64.41944 \\
-17.67815\end{array}$ & $\begin{array}{l}-122 \pm 2 \\
-141 \pm 2 \\
24 \pm \ldots \\
130 \pm \ldots \\
-79 \pm 1\end{array}$ & $\begin{array}{r}945 \pm 40 \\
950 \pm 50 \\
800 \pm 40 \\
870 \pm 60 \\
1060 \pm 100\end{array}$ & $\begin{array}{r}802 \\
940 \\
953 \\
1068 \\
1152\end{array}$ \\
\hline
\end{tabular}

Note. - The sample of outer Local Group galaxies used in the modeling of Section 2.3. Column (1) lists the galaxy name and column (2) its type. Columns (3) and (4) give the position on the sky. Column (5) lists the heliocentric line-of-sight velocity and, where available, its error. Velocities and sky positions (RA,DEC) were obtained from the NASA Extragalactic Database (NED). Column (6) lists the heliocentric distance and its error, from Grebel et al. (2003). Column (7) lists the Local Group barycentric distance $D_{\text {bary }}$, calculated assuming the M31 distance listed in Section 3.1 and a mass fraction $f_{\mathrm{M} 31}=0.53$. The galaxies in the table are sorted by their value of $D_{\text {bary }}$.

parts of the Local Group) compiled by Grebel, Gallagher, \& Harbeck (2003). From their table 1 we removed all galaxies listed as being (potentially) part of the Milky Way or M31 subgroups. We obtained the heliocentric line-of-sight velocities of the galaxies from the NASA Extragalactic Database (NED). We removed the Cetus dwarf from the sample, since it has no line-of-sight velocity available. We restricted our primary sample to galaxies with a Local Group barycentric distance less than $\sim 1.2 \mathrm{Mpc}$. The resulting sample consists of 5 galaxies, which are listed in Table 2, With this sample the analysis yields for M31: $v_{\mathrm{sys}}=-405 \pm 114 \mathrm{~km} \mathrm{~s}^{-1}$, $v_{W}=-126 \pm 63 \mathrm{~km} \mathrm{~s}^{-1}, v_{N}=-89 \pm 50 \mathrm{~km} \mathrm{~s}^{-1}$ (see also Table 3 below). The fit yields an estimate $\sigma=22 \mathrm{~km} \mathrm{~s}^{-1}$ for the one-dimensional dispersion of the galaxies around the space motion of the Local-Group barycenter. The small value of this dispersion is due to the fact that the sample galaxies reside at an average Local Group barycentric distance of $0.98 \mathrm{Mpc}$, which is consistent with the Local Group turn-around radius (e.g., Karachentsev et al. 2002), where the velocity with respect to the Local Group barycenter is zero by definition.

When comparing the analyses in Section 2.1 and the present section, there is an important difference in the expected galaxy velocities. The velocities of satellites around M31 are virialized, so the expectation value of a satellite velocity with respect to M31 is zero, independent of where the satellite is located on the sky. By contrast, the motions of the outer Local-Group galaxies around the Local Group barycenter are not virialized. Therefore, the expectation value of a galaxy velocity with respect to the Local Group barycenter is zero only if the galaxy is near the turn-around radius. If this is not the case, then equation (77) is valid only if the galaxies are distributed homogeneously around the Local Group. In reality, the distribution is both non-homogeneous and the number of galaxies is small. Therefore, addition to the sample of galaxies beyond the turn-around radius is expected to add both bias and shot-noise to the estimate of the M31 velocity. Moreover, galaxies significantly outside the turn-around radius do not necessarily need to follow the Local Group barycenter motion.

The sample in Table 2 consists of a rather small number of galaxies. So despite the aforementioned disadvantages, we did study the effect of adding more Local Group galaxies at larger barycentric distances. In particular, we tried to add the only other 6 Local Group galaxies within $2 \mathrm{Mpc}$ (namely: NGC 3109, Antlia, Sextans A and B, IC 5152 and KKR 25; Grebel et al. 2003) that are not believed to be associated with any other nearby structures. These reside at barycentric distances of 1.6-1.9 Mpc. Analysis of the combined sample of 11 galaxies yields for M31: $v_{\mathrm{sys}}=-608 \pm 154 \mathrm{~km} \mathrm{~s}^{-1}$, $v_{W}=-82 \pm 138 \mathrm{~km} \mathrm{~s}^{-1}, v_{N}=-46 \pm 82 \mathrm{~km} \mathrm{~s}^{-1}$. The fit yields an estimate $\sigma=50 \mathrm{~km} \mathrm{~s}^{-1}$ for the one-dimensional dispersion of the galaxies around the space motion of the Local-Group barycenter. The large deviation of $v_{\text {sys }}$ from the observed value as well as the increased $\sigma$ and formal errors support our assertion that adding these distant galaxies decreases the quality of the results. Nonetheless, the results for $v_{W}$ and $v_{N}$ are consistent with those inferred from the smaller sample, within the errors. So we conclude that the results for the M31 transverse motion are quite robust, and not very sensitive to the composition of the sample. This is further supported by the fact that our results are consistent within the errors with the preferred solutions obtained by Einasto \& LyndenBell (1982), despite their use of a sample that is only partially overlapping with ours.

\subsection{Comparison and Combination of Constraints}

The $v_{W}$ and $v_{N}$ for M31 inferred from the different methods and listed in Table 3 are shown in Figure 2 as colored data points with error bars. The weighted averages of all four of the independent estimates are $\left\langle v_{W}\right\rangle=-78 \pm 41 \mathrm{~km} \mathrm{~s}^{-1}$ and $\left\langle v_{N}\right\rangle=-38 \pm 34 \mathrm{~km} \mathrm{~s}^{-1}$. This is shown in the figure as a black data point with error bars.

The $\chi^{2}$ that measures the residuals between the individual measurements in Table 3 and the weighted averages is $\chi^{2}=8.1$ for $N=9$ degrees of freedom (12 measurements minus 3 parameters). Therefore, the re- 
TABLE 3

M31 Heliocentric Velocity Estimates

\begin{tabular}{llll}
\hline \hline \multicolumn{1}{c}{ Method } & $\begin{array}{c}v_{\text {sys }} \\
\mathrm{km} \mathrm{s}^{-1} \\
(1)\end{array}$ & $\begin{array}{c}v_{W} \\
\mathrm{~km} \mathrm{~s}^{-1} \\
(3)\end{array}$ & $\begin{array}{c}v_{N} \\
\mathrm{~km} \mathrm{~s}^{-1} \\
(4)\end{array}$ \\
\hline M31 Satels. & $-270 \pm 19$ & $-136 \pm 148$ & $-5 \pm 75$ \\
M33 PM & $-183 \pm 76$ & $-48 \pm 80$ & $71 \pm 84$ \\
IC 10 PM & $-346 \pm 76$ & $-16 \pm 80$ & $-47 \pm 81$ \\
Outer LG Gals. & $-405 \pm 114$ & $-126 \pm 63$ & $-89 \pm 50$ \\
\hline Weighted Av. & $-273 \pm 18$ & $-78 \pm 41$ & $-38 \pm 34$ \\
\hline
\end{tabular}

Note. - Estimates of the heliocentric velocity of M31 estimated using different methods, as indicated in column (1). The method based on the M31 satellite ensemble line-of-sight velocities is described in Section 2.1 and that based on the observed proper motions (PMs) of M33 and IC 10 is described in Section 2.2 and that based on the line-of-sight velocities of the satellites in outer regions of the Local Group is described in Section 2.3. Column (2) lists the estimated M31 systemtic line-of-sight velocities. Columns (3) and (4) lists the estimated M31 transverse velocities in the West and North directions, respectively. The bottom line of the table lists the weighted average of the results from the different methods.

sults for the $v_{W}$ and $v_{N}$ from the different methods are consistent within the errors. Among other things, this implies that there is no evidence that the dispersion of the peculiar velocities of M31 satellites in the transverse direction, which enters into the analysis of Section 2.2 , is larger than the value $\sigma=76 \mathrm{~km} \mathrm{~s}^{-1}$ derived from lineof-sight velocities in Section 2.1 This is consistent with the assumption that was made about the isotropy of the peculiar velocities.

The inferred weighted average of the three independent systemic velocity estimates is $\left\langle v_{\text {sys }}\right\rangle=-273 \pm 18 \mathrm{~km} \mathrm{~s}^{-1}$. This differs at the $1.6 \sigma$ level from the observed M31 velocity $v_{\mathrm{sys}}=-301 \pm 1 \mathrm{~km} \mathrm{~s}^{-1}$ (Courteau \& van den Bergh 1999), but the agreement in an absolute sense is better than the formal uncertainties in $\left(\left\langle v_{W}\right\rangle,\left\langle v_{N}\right\rangle\right)$. So this comparison provides no reason to mistrust our assumptions that the M31 satellites (Sections 2.1 and 2.2) and the outer Local Group galaxies (Section 2.3) move on average through space with the same velocity as M31 and the Local Group barycenter, respectively (see LyndenBell 1999 for an earlier discussion of this).

The analyses in the previous sections make assumptions about the velocity distributions of the satellites, but not about their spatial distributions. These spatial distributions are known to be inhomogeneous. In the case of the M31 subgroup, there are more satellites on the near side of M31 (i.e., between M31 and the Milky Way) than on the far side (e.g., McConnachie \& Irwin 2006). Moreover, recent studies have suggested that (some of) the satellites are concentrated near a plane surrounding M31 (e.g., Koch \& Grebel 2006). These facts by themselves do not affect our analysis at all, as long as the velocity distributions remain isotropic. However, the analysis would be affected if the satellite ensemble possessed a mean rotation. This is possible in some of the scenarios that have been suggested for a possible disk-like distribution of satellites (but not necessarily the favored scenarios; see, e.g., Metz, Kroupa, \& Jerjen 2007). In the method

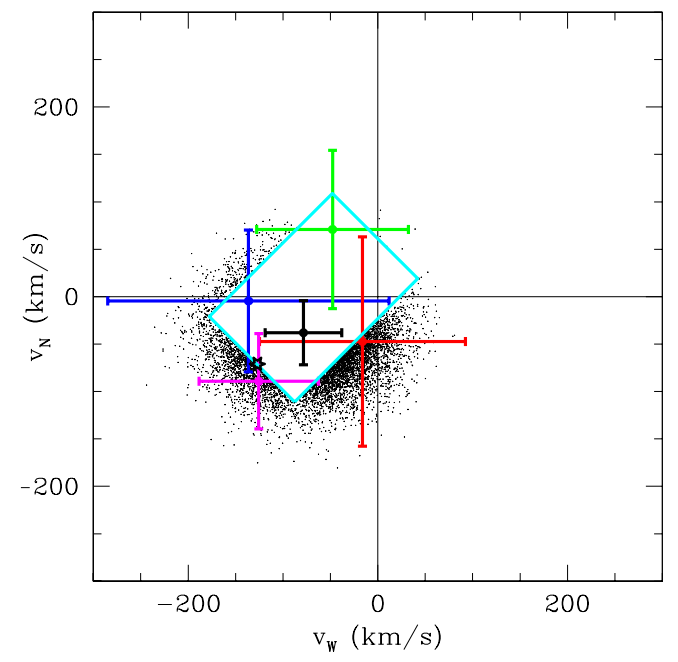

FIG. 2.- Estimates of the M31 heliocentric transverse velocity in the West and North directions. Data points with error bars are from Table 3 , based on the M31 satellite ensemble line-of-sight velocities (blue), the proper motion of M33 (green), the proper motion of IC 10 (red), the line-of-sight velocities of outer Local group satellites (magenta), or the weighted average of these results (black). The starred symbol indicates the transverse velocity that corresponds to a radial orbit for M31 with respect to the Milky Way. The cyan rectangle approximates the region that is ruled out in all of the theoretical models explored by Loeb et al. (2005), because the resulting relative orbit of M31 and M33 would have produced more disruption of the M33 disk than is observed. The small dots are the $18 \%$ of 30,000 samplings from the error ellipse belonging to the black data point that are consistent with the theoretical constraint.

of Section 2.1 we fit an apparent solid-body rotation field (eq. 3]) to the observed satellite velocities. Any intrinsic rotation would therefore bias the inferred transverse velocity. However, if this had been the case then we might have expected the M31 transverse velocity results of this method to be inconsistent with the results from the other methods that we have used, and in particular the method of Section 2.3 based on outer Local Group galaxies. The fact that the results from the different methods are actually statistically consistent therefore suggests that our results are not affected by potential rotation of the M31 satellite system.

In the comparisons of the different estimates listed in Table 3 it should be noted that they are not completely independent, because the line-of-sight velocities of M33 and IC10 enter not only in the analysis of Section 2.2. but also in that of Section 2.1. However, this is not a very important issue. We verified that if M33 and IC10 are removed from the line-of-sight velocity analysis in Section 2.1, then the changes in the results are well within the uncertainties.

\section{ORBITS}

\subsection{M31-Milky Way Orbit}

To calculate the velocity of M31 in the Galactocentric rest frame we adopt the same Cartesian coordinate system $(X, Y, Z)$ as in Section 2.3. We adopt a distance $D=770 \pm 40 \mathrm{kpc}$ for M31 (Holland 1998; Joshi et al. 2003; Walker 2003; Brown et al. 2004; McConnachie et al. 2005; Ribas et al. 2005). The position of M31 is then $\vec{r}=(-379.2,612.7,-283.1) \mathrm{kpc}$. To calculate the reflex motion of the Sun at the position of M31 we use 
the same solar velocity as in Section 2.3 and we use the standard IAU value $R_{0}=8.5 \mathrm{kpc}$ for the distance of the Sun from the Galactic Center (Kerr \& Lynden-Bell 1986), to which we assign an uncertainty of $0.5 \mathrm{kpc}$ (none of our results depend sensitively on this quantity). The velocity of the Sun then projects to $\left(v_{\text {sys }}, v_{W}, v_{N}\right)_{\odot}=$ $(172,128,71) \mathrm{kms}^{-1}$ at the position of M31. Since one observes the reflex of this, these values must be added to the observed M31 velocities to obtain its velocity in the Galactocentric rest frame. The velocity vector corresponding to the observed velocity component $v_{\text {sys }}$ given in Table 1 and the inferred $\left(\left\langle v_{W}\right\rangle,\left\langle v_{N}\right\rangle\right)$ given in Table 3 is then $\vec{V}_{\text {obs }}=(97 \pm 35,-67 \pm 26,80 \pm 32) \mathrm{km} \mathrm{s}^{-1}$. The errors (which are correlated between the different components) were obtained by propagation of the errors in the individual position and velocity quantities (including those for the Sun) using a Monte-Carlo scheme.

If the transverse velocity of M31 in the Galactocentric rest frame, $V_{\text {tan }}$, equals zero, then M31 moves straight towards the Milky Way on a purely radial (head-on collision) orbit. This orbit has $\left(v_{W}, v_{N}\right)_{\mathrm{rad}}=$ $(-127,-71) \mathrm{km} \mathrm{s}^{-1}$ (this is approximately the reflex of the velocity of the Sun quoted above, because the lines from the Sun to M31 and from the Galactic Center to M31 are almost parallel). The radial orbit is indicated as a starred symbol in Figure 2. The velocity $\vec{V}_{\text {obs }}$ calculated in the previous paragraph has tangential and radial components $V_{\text {tan,obs }}=59 \mathrm{~km} \mathrm{~s}^{-1}$ and $V_{\text {rad,obs }}=$ $-130 \mathrm{~km} \mathrm{~s}^{-1}$. The total velocity is $\left|\vec{V}_{\text {obs }}\right|=142 \mathrm{~km} \mathrm{~s}^{-1}$.

The value $V_{\text {rad,obs }}$ is an unbiased estimator of the true radial velocity $V_{\text {rad }}$. The associated uncertainty can be calculated in straightforward fashion using the previously described Monte-Carlo scheme. This yields $V_{\text {rad }}=-130 \pm 8 \mathrm{kms}^{-1}$. The uncertainty is due mostly to the uncertainty in our knowledge of the circular velocity of the LSR in the Galactic plane. The values of $V_{\text {tan,obs }}$ and $\left|\vec{V}_{\text {obs }}\right|$ are more difficult to interpret, because they are not unbiased estimators of the true velocities $V_{\tan }$ and $|\vec{V}|$. This is because the area coverage of $V_{\tan }$ values in the $\left(v_{W}, v_{N}\right)$ plane scales as $2 \pi V_{\tan } d V_{\mathrm{tan}}$, which produces a bias in the sense that any measurement error in $\left(v_{W}, v_{N}\right)$ tends to yield overestimates of $V_{\tan }$ and $|\vec{V}|$. To quantify and correct these biases we used Bayes' theorem, which yields the identity

$$
P\left(V_{\mathrm{tan}} \mid V_{\mathrm{tan}, \mathrm{obs}}\right) \propto P\left(V_{\mathrm{tan}}\right) P\left(V_{\mathrm{tan}, \mathrm{obs}} \mid V_{\mathrm{tan}}\right)
$$

We are interested in the quantity on the left-hand side, which is the probability distribution of $V_{\mathrm{tan}}$, given our measurement. The quantity $P\left(V_{\mathrm{tan}}\right)$ on the right-hand side is the Bayesian prior probability of $V_{\tan }$, which we assume to be flat (i.e., homogeneous). The quantity $P\left(V_{\mathrm{tan}, \mathrm{obs}} \mid V_{\mathrm{tan}}\right)$ is the probability of measuring a value $V_{\mathrm{tan}, \mathrm{obs}}$ if the actual value is $V_{\mathrm{tan}}$. This latter distribution is easily calculated using Monte-Carlo drawings, because the measurement uncertainties are known. Once the distributions $P\left(V_{\mathrm{tan}, \mathrm{obs}} \mid V_{\mathrm{tan}}\right)$ have been pre-calculated for all $V_{\mathrm{tan}}$, it is straightforward to obtain a Monte-Carlo sampling of the probability distribution $P\left(V_{\mathrm{tan}} \mid V_{\mathrm{tan}, \mathrm{obs}}\right)$. To this end one draws a random deviate $V_{\tan }$, and then accepts this value with probability $P\left(V_{\text {tan,obs }} \mid V_{\text {tan }}\right)$. The top left panel of Figure 3 shows the probability distribution $P\left(V_{\tan } \mid V_{\text {tan,obs }}\right)$ thus obtained. The median
$V_{\tan }=42 \mathrm{kms}^{-1}$. The fact that this is smaller than $V_{\text {tan,obs }}=59 \mathrm{~km} \mathrm{~s}^{-1}$ quantifies the aforementioned bias. The $1 \sigma$ confidence interval is $V_{\text {tan }} \leq 56 \mathrm{~km} \mathrm{~s}^{-1}$. So the radial orbit is consistent with the data at this confidence level. These results are discussed in the context of previous model predictions in Section 4.1. When combined with the value for $V_{\text {rad }}$, the $1 \sigma$ confidence interval around the median for the total velocity is $|\vec{V}|=138_{-11}^{+14} \mathrm{~km} \mathrm{~s}^{-1}$.

To get insight into the relative orbit of $\mathrm{M} 31$ and the Milky Way we assume that they can be approximated as point masses of mass $M_{\mathrm{M} 31}$ and $M_{\mathrm{MW}}$, respectively. In the center-of-mass frame, their orbit then has energy $E=\frac{1}{2} \mu|\vec{V}|^{2}-G \mu M /|\vec{r}|$ and angular momentum $L=\mu|\vec{r} \times \vec{V}|$, where the total mass is $M=M_{\mathrm{M} 31}+M_{\mathrm{MW}}$ and the reduced mass is $\mu=M_{\mathrm{M} 31} M_{\mathrm{MW}} / M$. The galaxies are bound to each other $(E<0)$ if $M \geq M_{\text {bind,min }}=$ $|\vec{r}||\vec{V}|^{2} / 2 G$. The orbit of the separation vector $\vec{r}$ is then a Kepler ellipse with eccentricity $e^{2}=1+\left(2 E L^{2} / G^{2} M^{2} \mu^{3}\right)$ and semi-major axis length $a=L^{2} /\left[G M \mu^{2}\left(1-e^{2}\right)\right]$. The pericenter separation is $r_{\text {peri }}=a /(1-e)$. The period is $T=2 \pi\left(a^{3} / G M\right)^{1 / 2}$. The orbit can be parameterized using the eccentric anomaly $\eta$ as (e.g., Kibble 1985; Binney \& Tremaine 1987)

$$
\begin{aligned}
& r=a(1-e \cos \eta), \\
& t=\left(a^{3} / G M\right)^{1 / 2}(\eta-e \sin \eta) .
\end{aligned}
$$

In this parameterization $\eta=0$ corresponds to the pericenter passage at $t=0$, while $\eta=2 \pi$ corresponds to the next pericenter passage at $t=T$. The radial and tangential velocities can be similarly parameterized as (e.g., Kochanek 1996)

$$
\begin{aligned}
& V_{\mathrm{rad}}=(G M / a)^{1 / 2}(e \sin \eta) /(1-e \cos \eta), \\
& V_{\mathrm{tan}}=(G M / a)^{1 / 2}\left(1-e^{2}\right)^{1 / 2} /(1-e \cos \eta) .
\end{aligned}
$$

It is generally assumed that the Local Group is a bound system that, due to its overdensity, decoupled from the Hubble expansion at fairly high redshift. Since then the orbital evolution of M31 and the Milky Way has been governed by Newtonian dynamics. Given this scenario and the orbital description given by equation (10), the Big Bang must have corresponded to a previous pericenter passage, which we can take to be $t=0$. The current time $t$ then corresponds to the age of the Universe, which has been tightly constrained using data from the Wilkinson Microwave Anisotropy Probe to be $t=13.73_{-0.15}^{+0.16} \mathrm{Gyr}$ (Spergel et al. 2007). With measurements of the current Galactocentric distance $r$ and velocities $V_{\text {rad }}$ and $V_{\text {tan }}$, the equations (10) and (111) can be solved for the quantities $M, a, e$ and $\eta$. The quantity $\eta$ must be in the interval $[\pi, 2 \pi]$ (so that M31 and the Milky Way are falling towards each other for the first time), since unplausibly high masses $M$ are otherwise required.

This methodology for modeling the Local Group is commonly called the "timing-argument". It has been widely applied and discussed in the literature (e.g., Kahn \& Woltjer 1959; Lynden-Bell 1981, 1999; Einasto \& Lynden-Bell 1982; Raychaudhury \& Lynden-Bell 1989; Kroeker \& Carlberg 1991; Kochanek 1996; Loeb et al. 2005), mostly to obtain joint estimates for the age of the Universe and the mass of the Local Group, often 

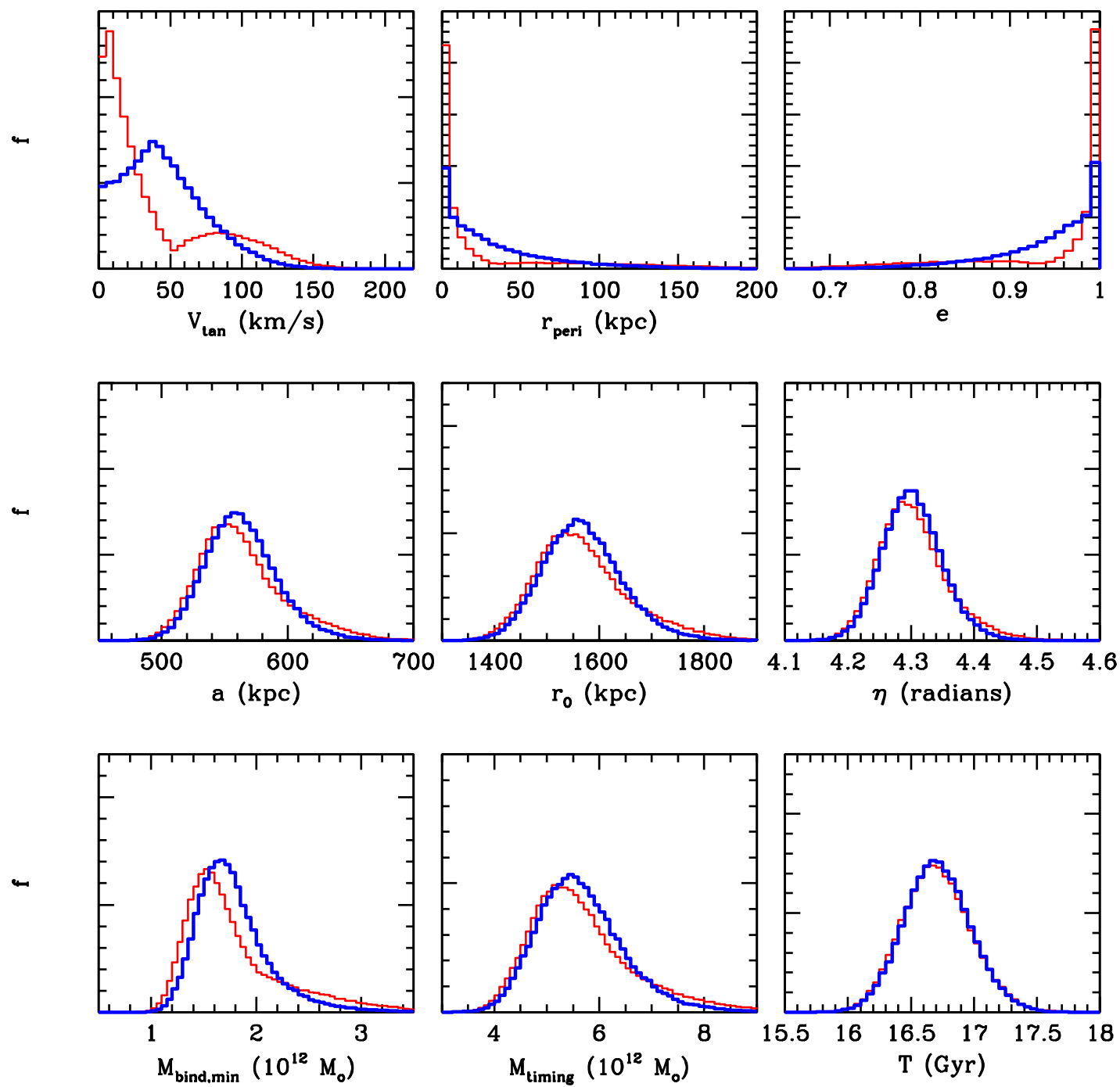

FIG. 3.- Probability histograms of: M31 Galactocentric tangential velocity $V_{\text {tan }}$; minimum total mass $M_{\text {bind,min }}$ of M31 and the Milky Way if the galaxies are bound; and assuming a bound orbit and the timing argument, the M31-Milky Way orbital pericenter distance $r_{\text {peri, }}$ orbital eccentricity $e$, orbital semi-major axis length $a$, orbital period $T$, current eccentric anomaly $\eta$, total mass $M_{\text {timing, }}$, and implied turnaround radius $r_{0}$ for test-particles on radial orbits around the M31-Milky Way system. The probability distributions were obtain using Monte-Carlo simulations as described in the text. The vertical scales are arbitrary. The blue curves take into account all observational uncertainties in the distances and velocities of both M31 and the Sun in the Galactocentric rest frame, as well as the observational uncertainties in the age of the Universe. The red curves also enforce the theoretical exclusion zone of Loeb et al. 2005 (cyan region in Figure 2), within which more tidal deformation of M33 would have been expected than is observed.

assuming a purely radial orbit. We can now do a more accurate analysis, both because we have an observational estimate of $V_{\tan }$, and because the age of the Universe can be assumed to be well-known from independent data. We have used this methodology on our results. Monte-Carlo error propagation was performed as described above, to obtain full probability distributions of $M, a, e, \eta, T$, and $r_{\text {peri }}$. The results are shown as histograms in Figure 3. The inferred $1 \sigma$ confidence intervals around the median are: $M=5.58_{-0.72}^{+0.85} \times 10^{12} \mathrm{M}_{\odot} ; a=561_{-26}^{+29} \mathrm{kpc}$; $\eta=4.301_{-0.045}^{+0.047}$ radians; $r_{\text {peri }}=23 \mathrm{kpc}$, with $1 \sigma$ confidence interval $r_{\text {peri }} \leq 40.9 \mathrm{kpc} ; T=16.70_{-0.26}^{+0.27} \mathrm{Gyr}$; and $e=0.959$, with $1 \sigma$ confidence interval $(1-e) \leq 0.072$. The implications of this result for $M$ are discussed in Section 4.2. The uncertainties in the listed quantities are due primarily to the uncertainties in $V_{\tan }$ and the M31 distance $D$. The uncertainties in these quantities con- tribute more or less equally to the uncertainties in $M, a$, $T$, and $\eta$. The uncertainties in the distance have little effect on $e$ and $r_{\text {peri }}$. All quantities vary monotonically with $V_{\tan }$ and $D$. Larger values of $V_{\tan }$ yield larger values of $M, a, \eta, T$, and $r_{\text {peri }}$, and smaller values of $e$. Larger values of $D$ yield larger values of $M, a, e, T$, and $r_{\text {peri }}$, and smaller values of $\eta$. Figure 3 also shows the probability distribution of $M_{\text {bind,min }}$, the minimum mass required for a bound orbit. The inferred $1 \sigma$ confidence intervals around its median is $M_{\text {bind,min }}=1.72_{-0.25}^{+0.26} \times 10^{12} \mathrm{M}_{\odot}$.

The anticipated collision between M31 and the Milky Way will happen at the next orbital pericenter, which in the Kepler model is at $T-t \approx 3.0 \pm 0.3 \mathrm{Gyr}$ from now. The orbital pericenter distance is much smaller than the sizes of the galaxies' dark halos. If the galaxies are assumed to have a logarithmic potential that reproduces the observed rotation curve amplitudes, then their radial extents $r_{t}$ are $235\left(M_{\mathrm{M} 31} / 3.4 \times 10^{12} \mathrm{M}_{\odot}\right) \mathrm{kpc}$ 
and $207\left(M_{\mathrm{MW}} / 2.3 \times 10^{12} \mathrm{M}_{\odot}\right) \mathrm{kpc}$, respectively (Loeb et al. 2005). The dark halos will intersect once the orbital separation becomes smaller than the sums of these sizes. This will have two consequences. First, the orbits will deviate from Kepler ellipses in the sense that the orbit will have less curvature and larger $r_{\text {peri }}$ than indicated by the previously derived Kepler orbit. Second, there will be dynamical friction, which will tend to increase the curvature and will tend to decrease $r_{\text {peri }}$. More complicated calculations are necessary to properly calculate the orbit once the dark halos of the galaxies start to overlap and to study the properties of the resulting merger. Such calculations were recently presented by Cox \& Loeb (2007). However, they adopted an orbit with $V_{\mathrm{tan}}=132 \mathrm{~km} \mathrm{~s}^{-1}$, $r_{\text {peri }}=450 \mathrm{kpc}$, and $e=0.494$. Comparison to the top panel of Figure 3 shows that this orbit is not consistent with our measurement of the M31 transverse velocity.

\subsection{M31-M33 Orbit}

Loeb et al. (2005; hereafter L05) recently derived a theoretical constraint on the transverse motion of M31 from the fact that M33 is relatively undisturbed. This appears to rule out orbits where M33 had a previous close interaction with M31. The exact shape of the region in $\left(v_{W}, v_{N}\right)$ space thus ruled out (being defined by L05 as: more than $20 \%$ of the M33 stars would have been stripped) has a complex shape and depends somewhat on the modeling assumptions, but it can be approximated by the solid cyan rectangle in Figure 2, This is an approximation to figure 2c of L05 (which quantified the transverse motion using Galactocentric rest-frame velocities $V_{\alpha \cos \delta}=-v_{W}-128 \mathrm{~km} \mathrm{~s}^{-1}$ and $\left.V_{\delta}=v_{N}+71 \mathrm{~km} \mathrm{~s}^{-1}\right)$. In some of their models (see their figures 2a,b) even a somewhat larger region is ruled out.

The overall shape of the excluded region in $\left(v_{W}, v_{N}\right)$ space can be understood with fairly simple calculations and arguments. In doing so, we ignore the observational uncertainties in the quantities of interest. This is sufficient for the scope of the present discussion, but should be included for more quantitative understanding of the M31-M33 orbit. Based on the data from Table 1 and the M33 and M31 distances from Sections 2.2 and 3.1, respectively, the distance between M33 and M31 is $203 \mathrm{kpc}$. The center of the excluded region in Figure2 is at $\left(v_{W}, v_{N}\right) \approx(-68,-11) \mathrm{km} \mathrm{s}^{-1}$. This defines the threedimensional velocity vector of M31, while for M33 that vector is known from Brunthaler et al. (2005). The radial and tangential velocity components of the separation vector are then $V_{\text {rad }}=-71 \mathrm{kms}^{-1}$ and $V_{\text {tan }}=126 \mathrm{~km} \mathrm{~s}^{-1}$. The Kepler orbit of the separation vector can be calculated similarly as in Section 3.1. In doing so, we assume that $M_{\mathrm{M} 31}=3.0 \times 10^{12}$, which is based on $M=5.58 \times 10^{12} \mathrm{M}_{\odot}($ Section 3.1$)$ and $f_{\mathrm{M} 31} \approx 0.54(\mathrm{com}-$ pare Section 2.3). We assume that the M33-to-M31 mass ratio is equal to the value $\sim(118 / 250)^{4}$ suggested by the Tully-Fisher relation and the galaxy's circular velocities (Corbelli \& Schneider 1997; Klypin et al. 2002). The orbit then has $a=120 \mathrm{kpc}, r_{\text {peri }}=27 \mathrm{kpc}, T=2.2 \mathrm{Gyr}$, and $e=0.77$. The value of $a$ is close to the minimum that this quantity can attain (i.e., maximum binding energy) as a function of $\left(v_{W}, v_{N}\right)$, which is $a_{\text {min }}=113 \mathrm{kpc}$. Given the value of $a$, M33 moves inside of the M31 dark halo for most of its orbit. Our assumption that all of the M31 mass resides at its center therefore overestimates the curvature of the orbit. The listed pericenter distance should thus be interpreted as a lower bound on the actual value. More detailed calculations, as in L05, are required to get a proper estimate of this quantity.

As one moves from $\left(v_{W}, v_{N}\right) \approx(-68,-11) \mathrm{kms}^{-1}$ along the diagonal that runs from the bottom left to the top right in Figure 2 2 the Kepler orbit value of $r_{\text {peri }}$ doesn't change much. However, $a$ increases, which means that the orbits become more eccentric and less bound (and ultimately unbound). The galaxies therefore spend less time in close vicinity of each other, and the relative velocity at pericenter increases. By contrast, as one moves from $\left(v_{W}, v_{N}\right) \approx(-68,-11) \mathrm{kms}^{-1}$ along the diagonal that runs from the top left to the bottom right in Figure 2, the Kepler orbit value of $e$ doesn't change much. But again, $a$ increases, which means that the orbits have larger pericenter separations and become less bound (and ultimately unbound). The energy dissipated during an encounter scales as $d E \propto 1 /\left(r^{4} V^{2}\right)$ in the impulsive tidal approximation (e.g., Binney \& Tremaine 1987, eq. [7$55]$ ), where $r$ is the impact parameter. Therefore, as one moves away along either diagonal in Figure 2, the structure of M33 will be less perturbed by the encounter. The excluded region in Figure 2 can therefore be understood to lowest order as the region where the orbital integral over $d E$ exceeds some threshold, with $r$ and $V$ calculated on the basis of the Kepler orbit.

Interestingly, the observationally implied transverse velocity of M31 from Table 3 falls right in the region for which considerable tidal deformation of M33 would have been expected. This M31 velocity yields a Kepler orbit for the M31-M33 separation vector with $a=127 \mathrm{kpc}$, $r_{\text {peri }}=30 \mathrm{kpc}, T=2.4 \mathrm{Gyr}$, and $e=0.76$. However, the uncertainties in the observationally implied velocities cannot be ignored. Upon performing Monte-Carlo sampling we find that as much as $18 \%$ of samplings from the error ellipse fall outside the cyan rectangle in Figure 2. and therefore do not violate the M33 tidal stripping argument. These $18 \%$ are shown as small black dots for a total sample of 30,000 drawings. The black dots can be viewed as a visual representation of the probability distribution of M31's transverse velocity obtained by taking into account not only the observational constraints derived here, but also the theoretical M33 stripping argument of L05. Since most of the dots fall close to the cyan rectangle, it is likely that there has been some tidal deformation of M33 by M31 (although not sufficient to pass L05's threshold for being considered excluded). This suggests that it will be worthwhile to perform deep searches for tidal tails and structures in the outer regions of M33, similar to those that have already been performed for M31 (e.g., Ferguson et al. 2002).

Our results for the relative M31-M33 orbit involve a small amount of circular reasoning, since we have assumed a priori that the residual space motion of M33 with respect M31 has the same dispersion $\left(\sigma=76 \mathrm{~km} \mathrm{~s}^{-1}\right.$ per coordinate, as derived from line-of-sight velocities in Section 2.1) as do the other M31 satellites. To avoid this circular reasoning one could ignore the result labeled "M33 PM" in Table 3 (green cross in Figure 2). The weighted average of the remaining M31 transverse motion estimates then becomes $\left\langle v_{W}\right\rangle=-89 \pm 47 \mathrm{kms}^{-1}$ and 
$\left\langle v_{N}\right\rangle=-60 \pm 37 \mathrm{~km} \mathrm{~s}^{-1}$. This differs by only $24 \mathrm{~km} \mathrm{~s}^{-1}$ from what we have used so far (last line of Table [3), and this difference is well within the uncertainties. This modified result still falls well inside the region excluded by L05. Therefore, our conclusions about the M31-M33 orbit are not influenced by the fact that we have used the M33 space velocity as one of the estimators of the M31 space velocity.

L05 only considered models with $M_{\mathrm{M} 31}=2.6-3.4 \times$ $10^{12} \mathrm{M}_{\odot}$. As is discussed in Section 4.2 below, it is possible that the M31 mass is actually lower than this. For example, Klypin et al. (2002) advocate $M_{\mathrm{M} 31}=$ $1.6 \times 10^{12} \mathrm{M}_{\odot}$. It follows both from the simple arguments of Section 3.2 and from the detailed calculations of L05 (their figure 2) that the amount of past tidal deformation of M33 is smaller for smaller values of $M_{\mathrm{M} 31}$. This would reduce the area of the excluded rectangular region in Figure 2, and would reduce the concern that our observational estimate of the M31 transverse velocity falls in the region of parameter space that was disfavored by L05. Also, the models of L05 (and hence the cyan region in Figure 2) do not account explicitly for the observational uncertainties in the assumed distances of M31 and M33. If the actual distances differ at the 1 or $2 \sigma$ level from the canonical values, then this could affect the location of the excluded region in transverse-velocity space.

It is straightforward to include the theoretical constraint of L05 into the M31-Milky Way timing argument calculations of Section 3.1. To address this, we applied the same Monte-Carlo scheme as in that section, but now with rejection of all Monte-Carlo drawn velocities with $\left(v_{W}, v_{N}\right)$ combinations in the region excluded by L05. This yields the probability histograms shown in red in Figure 3 . The distributions of $V_{\mathrm{tan}}, e$ and $r_{\text {peri }}$ become rather non-Gaussian, as can be easily understood from the distribution of points in Figure 2] However, the other distributions remain close to Gaussian, and are not very different from those obtained without using the LO5 M33 stripping argument. The same is true for the inferred $1 \sigma$ confidence intervals around the median values. For example, for the Local Group mass we obtain $M=5.50_{-0.76}^{+1.14} \times 10^{12} \mathrm{M}_{\odot}$, which is similar to the result of Section 3.1

\section{DISCUSSION}

\subsection{M31 Tangential Velocity}

Many previous studies of the M31-Milky Way system have assumed that their orbit can be approximated to be radial $\left(V_{\tan } \approx 0\right)$. This simplifies analyses based on the timing argument and, in the absence of a reliable $V_{\tan }$ measurement, was a reasonable guess based on simple cosmological arguments. In the absence of mutual gravitational interactions, peculiar velocities with respect to the Hubble flow decrease with time as $(1+z)$. In the M31-Milky Way system there is mutual gravitational attraction along the galaxy separation vector. This changes the radial velocity $V_{\text {rad }}$ from positive (receding) at high $z$ to negative (approaching) at the present time, as quantified by the timing argument. However, the angular momentum is conserved in a two-body system without external perturbations. Therefore, a significant presentday tangential motion in such as system implies an un- realistically high peculiar velocity at high redshift.

The situation is more complicated when the possibility of angular momentum exchange is taken into account. Tidal torques can lead to exchange between the spin angular momentum of the galaxies and their orbital angular momentum. This process may have contributed both to the observed spins of M31 and the Milky-Way, and to the tangential velocity component in their orbital motion (Gott \& Thuan 1978; Dunn \& Laflamme 1993). More importantly, tidal torques exerted by the galaxies outside of the Local Group also induce a tangential velocity component in the M31-Milky Way system (Raychaudhury \& Lynden-Bell 1989). A useful approach to study this effect, and more generally, the orbits of all the galaxies in the nearby Universe, is based on the principle of least action (Peebles 1989). This assumes that nearby galaxies arrived at their present configuration through gravitational interactions from a nearly homogeneous highredshift state with negligible peculiar velocities. Allowed solutions are those that minimize the relevant Hamiltonian action integral. This method can be used to fit observed galaxy velocities starting from their observed positions (Peebles et al. 1989; Peebles 1994), to fit observed galaxy distances starting from their observed velocities (Shaya, Peebles, \& Tully 1995; Schmoldt \& Saha 1998), or to fit observed galaxy velocities and distances simultaneously (Peebles et al. 2001, hereafter P01; Pasetto \& Chiosi 2007). The general prediction from the theoretical work that includes tidal torques is that M31 tangential velocities of $V_{\tan } \lesssim 200 \mathrm{~km} \mathrm{~s}^{-1}$ are expected in plausible models. ${ }^{1}$

Figure 6 of P01 shows the M31 transverse velocity vectors predicted in 30 minimum-action solutions for the nearby Universe. The reason that there are multiple possible solutions is due to the absence of observational knowledge of most galaxy proper motions. P01 characterized the M31 velocity using supergalactic angular coordinates in the Galactocentric rest frame. These are related to the heliocentric transverse velocity $v_{t}$ and the position angle $\Theta_{t}$ of the transverse motion on the sky, defined as in equation (2), according to

$$
\begin{aligned}
& v_{\mathrm{SGL}}=-v_{t} \cos \left(\Theta_{t}+34.60^{\circ}\right)-131 \mathrm{~km} \mathrm{~s}^{-1}, \\
& v_{\mathrm{SGB}}=v_{t} \sin \left(\Theta_{t}+34.60^{\circ}\right)-64 \mathrm{~km} \mathrm{~s}^{-1} .
\end{aligned}
$$

Our weighted average velocity $\left(\left\langle v_{W}\right\rangle,\left\langle v_{N}\right\rangle\right)$ given in Table 3 corresponds to $\left(v_{\mathrm{SGL}}, v_{\mathrm{SGB}}\right)=(-55,-21) \mathrm{km} \mathrm{s}^{-1}$. The $68.3 \%$ confidence ellipse around this measurement encloses 8 of the 30 viable solutions presented by P01. Our measurement is therefore fully consistent with their theoretical work. The action method is based on the assumptions that mass follows light and that the galaxy peculiar velocities are due to their mutual gravitational interactions. Our M31 transverse motion determination therefore provides no reason to doubt these assumptions (although cosmological N-body simulations sug-

\footnotetext{
1 The mass $M=M_{\mathrm{M} 31}+M_{\mathrm{MW}}$ calculated with the timing argument is a monotonically increasing function of $V_{\mathrm{tan}}$. So if one pre-assumes an upper limit to $M$, then one also obtains an upper limit to $V_{\mathrm{tan}}$. L05 used this approach to obtain $V_{\tan } \lesssim$ $120 \mathrm{kms}^{-1}$ based on the assumption that $M \leq 5.6 \times 10^{12} \mathrm{M}_{\odot}$. However, no physical motivation was provided for this assumed mass limit. Larger values of $V_{\text {tan }}$ are not inconsistent with the timing argument, but they do require higher masses.
} 
gest that these assumptions are at best only approximately satisfied; Martinez-Vaquero, Yepes, \& Hoffman 2007). The mass $M_{\mathrm{M} 31}+M_{\mathrm{MW}}$ assumed in the P01 models is $5.16 \times 10^{12} \mathrm{M}_{\odot}$, which is consistent with the range calculated in Figure 3 based on the timing argument. The mass was not varied independently in P01, but is within the factor $\sim 2$ range of masses for which the action method yields plausible solutions (Peebles et al. 1994). Pasetto \& Chiosi (2007) obtained a best-fit solution from their action modeling that corresponds to a heliocentric velocity $\left(v_{W}, v_{N}\right)=(-142,-41) \mathrm{km} \mathrm{s}^{-1}$. This value is near the edge of our $68.3 \%$ confidence ellipse, and is therefore also consistent with our measurement. The mass $M_{\mathrm{M} 31}+M_{\mathrm{MW}}$ assumed in their models is $5.36 \times 10^{12} \mathrm{M}_{\odot}$, which is also within the range calculated in Figure 3 based on the timing argument.

In summary, our measurement of the transverse velocity of M31 is consistent with the most recent theoretical models. Moreover, the fact that $73 \%$ of P01's actionmethod solutions do not fall within our $68.3 \%$ confidence ellipse suggests that our measurement has sufficient accuracy to provide meaningful constraints on the allowed solution space of the Local Group orbits and its history.

\subsection{Local Group Mass}

In Section 3.1 we calculated that the minimum $M=$ $M_{\mathrm{M} 31}+M_{\mathrm{MW}}$ required for a bound orbit is $M_{\mathrm{bind}, \min }=$ $1.72_{-0.25}^{+0.26} \times 10^{12} \mathrm{M}_{\odot}$ and that for a bound orbit the timing argument implies that $M=5.58_{-0.72}^{+0.85} \times 10^{12} \mathrm{M}_{\odot}$. These results are based on point mass dynamics, where the point masses are proxys for entire the Milky Way subgroup of galaxies and the entire M31 subgroup of galaxies (i.e., the parent galaxy plus its satellites), respectively. Only a negligible fraction of the light in the Local Group comes from galaxies that are not part of either of these subgroups (see Table 2). Therefore, the mass $M$ is a proxy for the total mass of the Local Group.

It is of interest to compare our mass estimates to those derived using independent arguments. Kochanek (1996) modeled the mass distribution of the Milky Way using equilibrium models for the velocities of Milky Way satellites, with the circular velocity of the disk and the escape velocity of stars in the solar neighborhood as additional constraints. This yielded a fairly well constrained mass of $(0.5 \pm 0.1) \times 10^{12} \mathrm{M}_{\odot}$ inside of $50 \mathrm{kpc}$. However, the total Milky Way mass depends on the extent of the dark halo, which is poorly constrained by any data. Kochanek's solutions therefore allowed total masses anywhere from $\sim 1-5 \times 10^{12} \mathrm{M}_{\odot}$ (see his figure 7 ). Wilkinson \& Evans (1999) obtained a similarly large range from their models, $M=1.9_{-1.7}^{+3.6} \times 10^{12} \mathrm{M}_{\odot}$, for the same reason. Both of these studies used rather arbitrarily parameterized density profiles. Klypin, Zhao \& Somerville (2002; hereafter K02) improved this situation by restricting the discussion to models with $\Lambda$ CDM cosmologically motivated density profiles and concentrations. This yielded a much more tightly constrained total mass. Their favored solution (their model A1) has a total virial mass of $1.0 \times 10^{12} \mathrm{M}_{\odot}$. The highest-mass model that is still consistent with the observational and theoretical constraints (their model A4; see also Besla et al. 2007) has a total virial mass of $2.0 \times 10^{12} \mathrm{M}_{\odot}$.

Evans \& Wilkinson (2000) and Evans et al. (2000) modeled the mass distribution of M31 using equilibrium models for the velocities of M31 satellites and halo tracers. This yielded $M=0.8_{-0.5}^{+1.6} \times 10^{12} \mathrm{M}_{\odot}$, suggesting that M31 be less massive than the Milky Way. However, as for the Milky Way modeling, the allowed mass range was large due to uncertainties in the dark halo extent. Again, K02 improved this situation by imposing theoretical constraints from $\Lambda$ CDM modeling. This yielded a favored model (their model B1) with a total virial mass of $1.6 \times 10^{12} \mathrm{M}_{\odot}$. The ratio $M_{\mathrm{M} 31} / M_{\mathrm{MW}}=1.6$ for the favored models in the K02 study (this implies $f_{\mathrm{M} 31}=0.62$, as defined in Section 2.3). This is similar to the value implied by the ratio of the galaxy's circular velocities and the Tully-Fisher relation, $\sim(250 / 220)^{4}=1.67$ (Einasto \& Lynden-Bell 1982).

K02 did not explore what maximum mass might still be consistent with the observational data for M31. However, to lowest order one may guess that the situation is similar as for the Milky Way, where the highest allowed mass is twice the favored mass. The maximum Local Group mass for the K02 models is therefore $\sim 5.2 \times 10^{12} \mathrm{M}_{\odot}$, while the favored mass is $M=2.6 \times 10^{12} \mathrm{M}_{\odot}$. This assumes that the derived virial masses of the Milky Way and Andromeda apply to their entire subgroups of satellites. This is reasonable, given the uncertainties already inherent in the estimates. The largest satellites of both subgroups (the Large Magellanic Cloud and M33, respectively) have $\lesssim 10 \%$ of the luminosity of their parent galaxy, and most other satellites contribute $\lesssim 1 \%$. Both the favored and the maximum mass of K02 exceed the value $M_{\text {bind,min }}=1.72_{-0.25}^{+0.26} \times 10^{12} \mathrm{M}_{\odot}$ derived in Section 3.1. This suggests that the Milky Way and M31 are indeed a bound system, as is usually assumed.

We showed that the timing argument for a bound M31Milky Way system implies a mass $M=5.58_{-0.72}^{+0.85} \times$ $10^{12} \mathrm{M}_{\odot}$. This is consistent with the maximum mass allowed by the K02 models, but not with the favored mass. Even the tail of the probability distribution for $M_{\text {timing }}$ in Figure 3 doesn't go as low as $M=2.6 \times 10^{12} \mathrm{M}_{\odot}$. The best agreements are obtained for the case of nearly radial orbits and a small M31 distance. For example, $V_{\text {tan }}=0$ and $D=700 \mathrm{kpc}$ yields $M_{\text {timing }}=4.4 \times 10^{12} \mathrm{M}_{\odot}$. But while this $V_{\tan }$ is consistent with our measurements, such a low distance is almost $2 \sigma$ away from the value $D=770 \pm 40 \mathrm{kpc}$ inferred using a wide range of methods (Holland 1998; Joshi et al. 2003; Walker 2003; Brown et al. 2004; McConnachie et al. 2005; Ribas et al. 2005).

The timing argument provides a rather simplistic view of the evolution of a binary galaxy in a cosmological context. Several authors have therefore quantitatively studied its accuracy. Models that address the gravitational influence and torques of structures outside of the Local Group have generally concluded that these do not significantly bias the mass estimates obtained from the timing argument (Raychaudhury \& Lynden-Bell 1989; Peebles et al. 1989, 2001; Pasetto \& Chiosi 2007; see also Section 4.1). However, these models generally treat individual galaxies as point masses. So this does not address the fact that the galaxies themselves assemble hierarchically over time.

To include hierarchical assembly it is necessary to perform more detailed dynamical calculations. Kroeker \& Carlberg (1991) studied the accuracy of the timing argu- 
ment by examining binary galaxies found in an $N$-body simulation of a closed CDM Universe. They found that when $V_{\text {tan }}$ is available, the timing argument provides an unbiased estimate of the total mass (measured in two spheres, centered on each galaxy, of radii one half the inter-galaxy separation). But estimates based on radial orbits yielded masses that were on average too low by a factor $\sim 1.7$. They also found that the galaxies must be bound for the timing argument to work, since it overestimates the actual mass of unbound pairs. The independent mass estimates of the Milky Way and M31 discussed above suggest that the galaxies are in fact bound, so this should not be an issue here. Kroeker \& Carlberg also show that the timing argument may not work well for pairs that are not isolated. This too should not be much of an issue for the M31-Milky Way system, given that the Sculptor, M81, and Maffei groups are all some $3 \mathrm{Mpc}$ away.

Goldberg (2001) reached somewhat different conclusions than Kroeker \& Carlberg. He modeled binary galaxies using a perturbative least action approach in which the galaxies are part of a continuous fluid that collapses over the course of the simulation. The results were cross-validated against an N-body simulation, with consistent results. From his calculations he concluded that the timing argument tends to overestimate the total mass of a pair by a factor of $\sim 2$. The reason for the discrepancy between this result and that of Kroeker \& Carlberg is unclear. However, Goldberg's calculations are quite idealized, while Kroeker \& Carlberg's calculations are now quite dated (both in terms of numerical sophistication and underlying cosmology). A more modern theoretical investigation of the accuracy of timing argument was therefore presented recently by Li \& White $(2008)^{2}$

$\mathrm{Li} \&$ White identified galaxy pairs in the so-called Millennium Simulation with properties similar to the Milky Way-M31 system. The high quality and detail of this cosmological simulation provides excellent statistics, with thousands of pairs available for study. They found that the radial-orbit timing mass estimator provides an unbiased estimate of the total mass of a pair (defined as the sum of the $M_{200}$ values, where $M_{200}$ is the mass inside the sphere over which the mean density is 200 times that of the Universe at large). With their preferred selection cuts, they found the ratio of the true to the estimated mass to be $A=0.99 \pm 0.41$, where we transformed their quoted interquartile range to a traditional Gaussian dispersion. The simulations do not confirm the finding of Kroeker \& Carlberg (1991) that the bias in the estimate depends on $V_{\text {tan }}$, but they do indicate that the dispersion in the estimate increases with $V_{\mathrm{tan}}$. Either way, the radial-orbit analysis of $\mathrm{Li} \&$ White is reasonable for the Milky Way-M31 system, since we have found that a radial orbit is consistent with the available observational constraints. The calculated "theoretical uncertainty" exceeds the uncertainties calculated here (see Figure 3) that result from propagation of observational errors. This theoretical uncertainty therefore drives the accuracy which we the Local Group mass is known from the timing argument. The preferred Local Group mass of

\footnotetext{
2 This paper came to our attention after the original submission of our own work.
}

K02 corresponds to a ratio $A=0.47$. This is $1.2 \sigma$ away from timing mass that we have calculated (with $\sigma$ being the quadrature sum of the theoretical and observational uncertainties). Therefore, the two results are quite consistent. K02 quote a mass uncertainty of a factor $\sim 2$, which is not too dissimilar from the timing mass uncertainty calculated by Li \& White (2008). Therefore, it is not a priori clear which estimate is to be preferred.

\subsection{Local Group Turn-Around Radius}

An independent mass estimate is obtained from the turn-around radius $r_{0}$ of the Local Group, defined as the distance from the Local Group barycenter at which galaxies have a radial velocity of zero (e.g., Lynden-Bell 1981). Using the same Keplerian formalism as in Section 3.1. galaxies on the turn-around surface have $\eta=\pi$ and $V_{\text {rad }}=0$. Equation (10) therefore gives that

$$
r_{0}=\left[G M(t / \pi)^{2}\right]^{1 / 3}(1+e),
$$

where, as before, $t$ is the age of the Universe and $r_{0}$ is usually evaluated for radial orbits $(e=1)$. Figure 3 shows the probability distribution of the predicted $r_{0}$ thus obtained, with $M$ taken from the timing argument. The inferred $1 \sigma$ confidence interval around the median is $r_{0}=1.56_{-0.07}^{+0.08} \mathrm{Mpc}$.

The $r_{0}$ predicted from the M31-Milky Way timing argument can be compared to the observationally inferred value, $r_{0}=0.94 \pm 0.10 \mathrm{Mpc}$ (e.g., Karachentsev et al. 2002). These values are not consistent. Since $r_{0} \propto$ $M^{1 / 3}$, the observed $r_{0}$ implies a Local Group mass of only $M=(1.3 \pm 0.3) \times 10^{12} \mathrm{M}_{\odot}$. Similar results are obtained when this method is applied to other nearby groups (Karachentsev et al. 2005). This mass is lower than that implied by the timing argument (as stressed previously by Lynden-Bell 1999) and it is also lower than that implied by almost all studies of the individual masses of the Milky Way and M31 (see Section 4.2). Moreover, comparison of this mass to $M_{\text {bind,min }}=1.72_{-0.25}^{+0.26} \times 10^{12} \mathrm{M}_{\odot}$ from Section 3.1 suggests that M31 and the Milky Way then do not form bound pair. This would make the Local Group little more than a chance superposition of two spiral galaxies. Such a configuration has fairly low a priori probability, given the local density of spiral galaxies (van den Bergh 1971).

The agreement between the observed and predicted turn-around radii improves if the galaxies defining the turn-around surface are not on radial orbits. A value $e=0.2$ in equation (13) would yield perfect agreement. However, the corresponding orbits are then nearly circular and this would produce a considerable thickness in the zero-velocity surface which is inconsistent with observations (Lynden-Bell 1999). So a more reasonable conclusion may be that mass estimates based on the turnaround radius are systematically biased towards low values. Direct evidence for this comes from the action modeling of, e.g., Peebles et al. (2001). Their models reproduce both the distances and redshifts of the galaxies in the outer regions of the Local Group (see Table 2), despite the use of a Local Group mass $M=5.16 \times 10^{12} \mathrm{M}_{\odot}$ that significantly exceeds the mass implied by equation (13). This suggests that the isolated collapse picture on which the concept of a turn-around surface is based may not be suitable for the Local Group. The under- 
lying reason may be that the dynamical structure near the edges of the Local Group is significantly influenced by structures in the mass distribution on larger scales. It should also be noted that the even in idealized models the zero-velocity surface is not actually expected to be a sphere, but an elongated ellipsoid (e.g., figure 6 in Peebles et al. 1989). This is not generally taken into account in observational determinations of $r_{0}$.

\section{CONCLUSIONS}

We estimated the transverse motion of M31 under the assumption that the satellites of M31 follow the motion of M31 through space, and under the assumption that the galaxies in the outer parts of the Local Group follow the motion of the Local Group barycenter through space. The first method was applied independently to a sample of 17 satellites with known line-of-sight velocities, and to the 2 satellites M33 and IC 10 with known proper motions. The second method was applied to a sample of 5 galaxies near the Local Group turn-around radius (and we showed that inclusion of 6 more distant galaxies does not significantly affect the results). The results from the different methods are mutually consistent and successfully recover the known line-of-sight velocity of M31. The weighted average heliocentric transverse velocity of M31 from the different methods in the West and North directions are found to be $\left\langle v_{W}\right\rangle=-78 \pm 41 \mathrm{~km} \mathrm{~s}^{-1}$ and $\left\langle v_{N}\right\rangle=-38 \pm 34 \mathrm{~km} \mathrm{~s}^{-1}$.

We used the known line-of-sight velocity with the newly inferred transverse velocity to determine the radial and tangential Galactocentric velocity components of M31. We used a Bayesian analysis to obtain the statistical probability distribution for $V_{\tan }$, properly corrected for observationally induced errors and biases. The results are that $V_{\mathrm{rad}}=-130 \pm 8 \mathrm{~km} \mathrm{~s}^{-1}$ and that the probability distribution for $V_{\tan }$ has a median value of $42 \mathrm{~km} \mathrm{~s}^{-1}$ and a $1 \sigma$ confidence interval $V_{\tan } \leq 56 \mathrm{~km} \mathrm{~s}^{-1}$. A purely radial orbit is consistent with the data at this confidence level. These velocities are consistent with the predictions of the most recent action-method models for the history of the Local Group, and have small enough errors that they can start to meaningfully constrain the full solution space allowed by these models.

We used the M31 velocity vector to constrain the relative M31-Milky Way orbit. The minimum mass required for a bound orbit is $M_{\text {bind,min }}=1.72_{-0.25}^{+0.26} \times 10^{12} \mathrm{M}_{\odot}$. If the orbit is indeed bound, then the timing argument combined with the known age of the Universe implies that $M=5.58_{-0.72}^{+0.85} \times 10^{12} \mathrm{M}_{\odot}$. This is consistent with mass range suggested by cosmologically motivated models for the individual structure and dynamics of M31 and the Milky Way, respectively. However, the timing mass is on the high end of the allowed range, with the most favored models in the study of K02 yielding a Local Group mass of only $2.6 \times 10^{12} \mathrm{M}_{\odot}$. This indicates that the M31 and the Milky Way are indeed bound, but that their total mass could be lower than suggested by the timing argument. Li \& White (2008) performed a theoretical calibration of the timing argument and found that while the timing argument mass is unbiased, it does have a theoretical dispersion of $41 \%$. Therefore, it is not statistically implausible that for the specific case of the Milky Way-M31 system the timing argument mass could be an overestimate by a factor of $\sim 2$. The method of estimating the Local Group mass based on the size of the turnaround (zero-velocity) radius yield systematically lower masses than other methods, and is in fact not consistent with a bound nature for the M31-Milky Way orbit. This and other arguments suggest that this method yields incorrect masses that are systematically biased low.

The M31 transverse velocity implies that M33 is in a tightly bound orbit around M31. The calculations of L05 indicate that for $82 \%$ of Monte-Carlo samplings from the error ellipse, more tidal deformation of M33 would have been expected than is observed. However, this assumes that $M_{\mathrm{M} 31}=2.6-3.4 \times 10^{12} \mathrm{M}_{\odot}$. The percentage is lower, and the agreement between observational and theoretical constraints on the M31 transverse velocity is better, if the M31 mass is as low as $1.6 \times 10^{12} \mathrm{M}_{\odot}$, as advocated by K02. Nonetheless, our results indicate that some tidal deformation of M33 could certainly have occurred. So it will be worthwhile to perform more deep searches for tidal tails and structures in the outer regions of M33. The estimates of the Local Group timing mass do not change significantly if the theoretical constraints from the L05 study of M33 structure are strictly enforced.

The transverse velocity inferred for M31 (the weighted average in Table 3) corresponds to a proper motion $\mu_{W} \equiv-\dot{\alpha} \cos \delta=-21.5 \pm 11.1 \mu \operatorname{as~yr}^{-1}$ and $\mu_{N} \equiv \dot{\delta}=$ $-10.4 \pm 9.3 \mu \operatorname{asyr}^{-1}$ at a distance $D=770 \mathrm{kpc}$. It will be difficult to obtain an actual proper motion measurement in the near future that will rival the accuracy of this. The most accurate Hubble Space Telescope proper motion measurements for any Local Group galaxy (for the Large and Small Magellanic Cloud; Kallivayalil et al. 2006a,b) have errors of $50-80 \mu \mathrm{as} \mathrm{yr}^{-1}$. VLBI measurements of water masers can provide errors of only $5-10 \mu \mathrm{as} \mathrm{yr}^{-1}$ (Brunthaler et al. 2005, 2007). This is actually not much better than what we have already presented here. Moreover, no water masers are known in M31 to which this technique can be applied and, at present detection limits, may be none should be expected (Brunthaler et al. 2006). Therefore, a high accuracy measurement of the proper motion of M31 will have to await future generations of astrometric satellites. However, even SIM or GAIA measurements will at best have uncertainties that are only a few times smaller than those reported here (see section 12 of Unwin et al. 2007), and moreover, results are not expected from these satellites for at least another decade.

It may be possible to improve the results presented here by using a larger number of tracers of the M31 velocity. If the sample of M31 satellites with water maser proper motions can be increased from 2 to $\sim 8$, then this would halve the uncertainty in the average result from the method in Section 2.2. However, this may not be possible. Water masers are often associated with regions of star formation, whereas most M31 satellites are early type dwarf ellipticals or spheroidals (see Table 1). Even in the gas-rich satellites the prospects for new water maser discoveries are not good (Brunthaler et al. 2006). Therefore, the number of satellites for which water maser proper motions can be obtained is fundamentally limited. Alternatively, it may be possible to increase the number of tracers at large distances from M31 for which lineof-sight velocities are available. A significant increase 
would be needed (from 17 to $\sim 68$ ) to merely halve the uncertainties in our result from Section 2.1. While new satellites of M31 continue to be discovered on a regular basis (e.g., Zucker et al. 2007; Ibata et al. 2007), this is unlikely to significantly affect the statistics. Individual Red Giant Branch stars in the outer halo of M31 may be more promising (e.g., Gilbert et al. 2006), since it is possible, at least in principle, to obtain line-of-sight velocities for large numbers of such stars.
We thank Nitya Kallivayalil and Gurtina Besla for a careful reading of this paper, and Anatoly Klypin, Frank van den Bosch, Lars Hernquist, and T.J. Cox for useful discussions. We thank the anonymous referee for helpful suggestions that improved the presentation of the paper. We made use of the NASA/IPAC Extragalactic Database (NED), which is operated by the Jet Propulsion Laboratory, Caltech.

\section{REFERENCES}

Barnard, E. E. 1917, AJ, 30, 175

Besla, G., Kallivayalil, N., Hernquist, L., Robertson, B., Cox, T J., van der Marel, R. P., \& Alcock, C. 2007, ApJ, 668, 949

Binney, J., \& Tremaine, S. 1987, Galactic Dynamics (Princeton: Princeton University Press)

Brown, T. M., Ferguson, H. C., Smith, E., Kimble, R. A. Sweigart, A. V., Renzini, A., \& Rich, R. M. 2004, AJ, 127, 2738

Brunthaler, A., Reid, M. J., Falcke, H., Greenhill, L. J., \& Henkel, C. 2005, Science, 307, 1440

Brunthaler, A., Henkel, C., de Blok, W. J. G., Reid, M. J., Greenhill, L. J., \& Falcke, H. 2006, A\&A, 457, 109

Brunthaler, A., Reid, M. J., Falcke, H., Henkel, C., \& Menten, K. M. 2007, A\&A, 462, 101

Chapman, S. C., Ibata, R., Lewis, G. F., Ferguson, A. M. N., Irwin, M., McConnachie, A., \& Tanvir, N. 2005, ApJ, 632, L87

Chapman, S. C., et al. 2007, ApJ, 662, L79

Corbelli, E. \& Schneider, S. E. 1997, ApJ, 479, 244

Courteau, S. \& van den Bergh, S. 1999, AJ, 118, 337

Cox, T. J., \& Loeb, A. 2007, MNRAS, submitted arXiv:0705.1170

Dehnen, W., Binney, J. J. 1998, MNRAS, 298, 387

Dunn, A. M., \& Laflamme, R. 1993, MNRAS, 264, 865

Einasto, J., \& Lynden-Bell, D. 1982, MNRAS, 199, 67

Evans, N. W., \& Wilkinson, M. I. 2000, MNRAS, 316, 929

Evans, N. W., Wilkinson, M. I., Guhathakurta, P., Grebel, E. K. \& Vogt, S. S. 2000, ApJ, 540, L9

Ferguson, A. M. N., Irwin, M. J., Ibata, R. A., Lewis, G. F., \& Tanvir, N. R. 2002, AJ, 124, 1452

Gilbert, K. M., et al. 2006, ApJ, 652, 1188

Goldberg, D. M. 2001, ApJ, 550, 87

Gott, J. R., \& Thuan, T. X. 1978, ApJ, 223, 426

Grebel, E. K., Gallagher III, J. S., \& Harbeck, D. 2003, AJ, 125, 1926

Holland, S. 1998, AJ, 115, 1916

Ibata, R., Martin, N. F., Irwin, M., Chapman, S., Ferguson, A. M. N., Lewis, G. F., McConnachie, A. W. 2007, ApJ, 671, 1591

Joshi, Y. C., Pandey, A. K., Narashima, D., Sagar, R., \& Giraud-Héraud, Y. 2003, A\&A, 402, 113

Kahn, F. D., \& Woltjer, L. 1959, ApJ, 130, 705

Kalirai, J. S., Guhathakurta, P., Zucker, D. B., et al. 2007, in prep.

Kallivayalil, N., van der Marel, R. P., Alcock, C., Axelrod, T., Cook, K. H., Drake, A. J., \& Geha, M. 2006a, ApJ, 638, 772

Kallivayalil, N., van der Marel, R. P., \& Alcock, C. 2006b, ApJ, 652,1213

Karachentsev, I. D. et al. 2002, A\&A, 389, 812

Karachentsev, I. D. 2005, AJ, 129, 178

Kerr, F. J., \& Lynden-Bell, D. 1986, MNRAS, 221, 1023

Kibble, T. W. B. 1985, Classical Mechanics (New York: Longman)

Klypin, A., Zhao., H. S., \& Somerville, R. S. 2002, 573, 597 (K02)

Koch, A., \& Grebel, E. K. 2006, AJ, 131, 1405

Kochanek, C. S. 1996, ApJ, 457, 228
Kroeker, T. L., \& Carlberg, R. G. 1991, ApJ, 376, 1

Li, Y.-S., \& White, S. D. M. 2008, MNRAS, in press arXiv:0710.3740

Loeb, A., Reid, M. J., Brunthaler, A., \& Falcke, H. 2005, ApJ, 633,894 (L05)

Lynden-Bell, D., \& Lin, D. N. C. 1977, MNRAS, 181, 37

Lynden-Bell, D. 1981, The Observatory, 101, 111

Lynden-Bell, D. 1999, in "The stellar content of Local Group galaxies", Proc. IAU Symp. 192, Whitelock, P., \& Cannon, R. eds., p. 39 (San Francisco: Astronomical Society of the Pacific)

Majewski, S. R., et al. 2007, ApJL, 670, L9

Martin, N. F., Ibata, R. A., Irwin, M. J., Chapman, S., Lewis, G. F., Ferguson, A. M. N., Tanvir, N., \& McConnachie, A. W. 2006, MNRAS, 371, 1983

Martinez-Vaquero, L. A., Yepes, G., \& Hoffman, Y. 2007, MNRAS, 378, 1601

McConnachie, A. W., Irwin, M. J., Ferguson, A. M. N., Ibata, R. A., Lewis, G. F., \& Tanvir, N. 2004, MNRAS, 350, 243

McConnachie, A. W., Irwin, M. J., Ferguson, A. M. N., Ibata, R. A., Lewis, G. F., \& Tanvir, N. 2005, MNRAS, 356, 979

McConnachie, A. W., \& Irwin, M. J. 2006, MNRAS, 365, 902

Metz, M., Kroupa, P., \& Jerjen, H. 2007, MNRAS, 374, 1125

Pasetto, S., \& Chiosi, C. 2007, A\&A, 463, 427

Peebles, P. J. E. 1989, ApJ, 344, L53

Peebles, P. J. E., Melott, A. L., Holmes, M. R., \& Jiang, L. R. 1989, ApJ, 345, 108

Peebles, P. J. E. 1994, ApJ, 429, 43

Peebles, P. J. E., Phelps, S. D., Shaya, E. J., \& Tully, R. B. 2001 ApJ, 554, 104 (P01)

Raychaudhury, S., \& Lynden-Bell, D. 1989, MNRAS, 240, 195

Ribas, I., Jordi, C., Vilardell, F., Fitzpatrick, E. L., Hilditch, R. W., \& Guinan, E. F. 2005, ApJ, 635, L37

Schmoldt, I. M., \& Saha, P. 1998, AJ, 115, 2231

Shaya, E. J., Peebles, P. J. E., \& Tully, R. B. 1995, ApJ, 454, 15

Spergel, D. N., et al. 2007, ApJS, 170, 377

Unwin, S. C. 2007, PASP, in press arXiv:0708.3953

van den Bergh, S. 1971, A\&A, 11, 154

van den Bergh, S. 2000, The Galaxies of the Local Group (Cambridge: Cambridge University Press)

van der Marel, R. P., Magorrian, J., Carlberg, R. G., Yee, H. K.

C., \& Ellingson, E. 2000, AJ, 119, 2038

van der Marel, R. P., \& Cioni, M.-R. 2001, AJ, 122, 1807

van der Marel, R. P., Alves, D. R., Hardy, E., \& Suntzeff, N. B. 2002, AJ, 124, 2639

Wilkinson, M. I., \& Evans, N. W. 1999, MNRAS, 310, 645

Walker, A. R. 2003, in Stellar Candles for the Extragalactic Distance Scale, D. Alloin \& W. Gieren, eds., 265 (New York: Springer)

Yahil, A., Tammann, G. A., \& Sandage, A. 1977, ApJ, 217, 903

Zucker, D., et al. 2007, ApJ, 659, L21 